\title{
Improved methods for simulating nearly extremal binary black holes
}

\author{
Mark A. Scheel, ${ }^{1}$ Matthew Giesler, ${ }^{1,2}$ Daniel A. Hemberger, ${ }^{1}$ Geoffrey Lovelace, ${ }^{2,1}$ \\ Kevin Kuper, ${ }^{2}$ Michael Boyle, ${ }^{3}$ Béla Szilágyi, ${ }^{1}$ and Lawrence E. Kidder ${ }^{3}$ \\ ${ }^{1}$ Theoretical Astrophysics 350-17, California Institute of Technology, Pasadena, CA 91125, USA \\ ${ }^{2}$ Gravitational Wave Physics and Astronomy Center, California State University Fullerton, Fullerton, California 92834, USA \\ ${ }^{3}$ Center for Radiophysics and Space Research, Cornell University, Ithaca, New York 14853, USA
}

(Dated: December 5, 2014)

\begin{abstract}
Astrophysical black holes could be nearly extremal (that is, rotating nearly as fast as possible); therefore, nearly extremal black holes could be among the binaries that current and future gravitational-wave observatories will detect. Predicting the gravitational waves emitted by merging black holes requires numerical-relativity simulations, but these simulations are especially challenging when one or both holes have mass $m$ and spin $S$ exceeding the Bowen-York limit of $S / m^{2}=0.93$. We present improved methods that enable us to simulate merging, nearly extremal black holes more robustly and more efficiently. We use these methods to simulate an unequal-mass, precessing binary black hole coalescence, where the larger black hole has $S / m^{2}=0.99$. We also use these methods to simulate a non-precessing binary black hole coalescence, where both black holes have $S / m^{2}=0.994$, nearly reaching the Novikov-Thorne upper bound for holes spun up by thin accretion disks. We demonstrate numerical convergence and estimate the numerical errors of the waveforms; we compare numerical waveforms from our simulations with post-Newtonian and effective-one-body waveforms; we compare the evolution of the black-hole masses and spins with analytic predictions; and we explore the effect of increasing spin magnitude on the orbital dynamics (the so-called "orbital hangup" effect).
\end{abstract}

\section{INTRODUCTION}

Second-generation interferometers such as Advanced LIGO, Virgo, and KAGRA [1-4] will soon begin searching for gravitational waves. To increase the number of gravitational-wave detections and to maximize what we can learn about the waves' sources, we require accurate theoretical models of the sources and the emitted gravitational radiation.

The inspiral, merger, and ringdown of binary black holes (BBHs) are among the most promising astrophysical sources of gravitational waves. As the black holes orbit, they lose energy to gravitational radiation, inspiraling until they collide and merge to form a final black hole (the "remnant") that eventually settles to a stationary Kerr state.

$\mathrm{A} \mathrm{BBH}$ is characterized by 7 intrinsic parameters: the spin angular momenta $\vec{S}$ of each hole and the mass ratio $q$. The spin magnitude of a black hole is often characterized by the dimensionless quantity $\chi \equiv S / m^{2}$, where $S=|\vec{S}|, m$ is the black hole mass, and we use geometrized units where $c=G=1$. A black hole with the maximum possible dimensionless spin $\chi=1$ is called extremal. There is considerable uncertainty in the expected mass ratios and spins of astrophysical BBHs that are likely to be detected by gravitational-wave interferometers; however, there is evidence that nearly extremal black holes exist in nature. For instance, recent measurements of stellar-mass black holes (such as Cygnus X-1 [5-7], GRS 1915+105 [8], and GX 339-4 [9]) and supermassive black holes (such as Swift J0501.9-3239 [10]) suggest that there could be a population of black holes with spins of $\chi \sim 1$. (See, e.g., Refs. [11, 12] for reviews of astrophysical blackhole spin measurements.)
Post-Newtonian (PN) methods accurately model the binary evolution and the emitted gravitational radiation during the early inspiral [13], but numerical simulations solving the full Einstein equations are necessary to model the binary through late inspiral, merger, and ringdown. Following breakthroughs in 2005-2006 [14-16], a number of research groups have made tremendous progress toward simulating merging black holes with different blackhole masses and spins (see, e.g., [17-20] for recent reviews), and several groups are building catalogs of $\mathrm{BBH}$ simulations [21-26].

So far, the region of the parameter space with black hole spins near the theoretical maximum $\chi=1$ remains almost completely unexplored. Numerical simulations of nearly extremal, merging black holes are especially challenging. One reason for this is that initial data for a $\mathrm{BBH}$ must satisfy the Einstein constraint equations, but the simplest method for constructing constraintsatisfying initial data, the Bowen-York method [27-29], cannot yield initial data with nearly extremal black holes. This is because the Bowen-York construction assumes that the initial spatial geometry is conformally flat (i.e., that the initial spatial metric is proportional to the metric of flat space). Conformally flat spacetimes cannot represent black holes that i) are in equilibrium, and ii) possess linear [30] or angular [31, 32] momentum; therefore, conformally-flat spinning black holes are out of equilibrium and will quickly relax to an equilibrium state. Specifically, Bowen-York puncture initial data can produce BBHs with initial spins as large as $\chi=0.984$, but when evolved, the spins rapidly relax to about $\chi=0.93$ or less (the Bowen-York limit) [33-35].

Even given initial data containing black holes with spins exceeding the Bowen-York limit, evolving those 
data through inspiral, merger, and ringdown is especially challenging for two reasons, as discussed in Refs. [3638]. First, the portion of the spacetime near the horizons requires very high resolution (and thus high computational cost), since metric gradients are much larger than for lower spins. Second, for evolution methods that excise the singularities inside each black hole and evolve only the exterior region, constructing and maintaining a suitable computational domain that keeps each excision boundary just inside the corresponding apparent horizon becomes more and more challenging as the spin approaches extremality.

In this paper, we use the phrase "nearly extremal" to refer to $\chi>0.93$, i.e., to a black hole with a spin above the Bowen-York limit. Note that a black hole with $\chi=0.93$ is significantly less extremal than a black hole with $\chi=0.998$, the Novikov-Thorne upper bound for black holes spun up by accretion [39, 40]. This is because the effects of spin scale nonlinearly with increasing $\chi$. For instance, if the rotational energy of a Kerr black hole with a fixed mass is denoted $E_{\text {rot }}(\chi)$, then $E_{\text {rot }}(0.93) / E_{\text {rot }}(1)$ is only $59 \%$, while $E_{\text {rot }}(0.998) / E_{\text {rot }}(1)$ is $92.5 \%$ (c.f., Fig. 1 of Ref. [36]). Furthermore, the total energy that a BBH emits in gravitational waves also scales nonlinearly with $\chi$. For example, for equal masses and equal spins aligned with the orbital angular momentum, a BBH with $\chi=1$ radiates $10 \%$ more energy than a $\mathrm{BBH}$ with $\chi=0.93$, whereas a $\mathrm{BBH}$ with $\chi=0.07$ radiates only $4 \%$ more energy than a BBH with $\chi=0$ (Eq. (9) of Ref. [41]). Nonlinear scaling with $\chi$ is also seen for binaries consisting of a black hole and a neutron star: the amount of neutron-star matter remaining outside the black hole just after tidal disruption increases very rapidly with black-hole spin (Fig. 10 of Ref. [38]).

Several groups have constructed and evolved BowenYork puncture initial data with spins near (but below) the Bowen-York limit [42-45]. Recently, Ruchlin et al. [46] constructed and evolved puncture initial data for a head-on collision of two black holes with equal mass and spins of magnitude $\chi=0.99$. Only four previously published simulations [24, 36, 37] out of hundreds published to date contain the quasi-circular coalescence of BBHs with $\chi>0.93$. These four simulations were evolved using the Spectral Einstein Code (SpEC) [47] from "superposed-Kerr-Schild" excision initial data [48] and have equal masses and equal spins aligned or antialigned with the orbital angular momentum.

In this paper, we present technical improvements that have enabled us to simulate BBHs with black-hole spins up to $\chi=0.994$ (i.e., $E_{\text {rot }}(0.994) / E_{\text {rot }}(1)=87.1 \%$ ) and to complete the first nearly extremal BBH simulation that includes precession. We compare the results of these simulations with analytical models describing the remnant properties (e.g. final spin and total radiated energy) as a function of the initial black hole spins; these models were constructed using lower-spin simulations and then extrapolated to higher spins. We measure the slow increase in mass ("tidal heating") and decrease in spin ("tidal torquing") of the individual black holes before merger, and we compare these measurements with perturbative calculations of the same quantities. We also compare gravitational waveforms from these simulations with post-Newtonian and effective-one-body [49] models.

The methods described here allow us to robustly explore the portion of the $\mathrm{BBH}$ parameter space where one or both black holes are nearly extremal. Simulations using these methods will enable us to calibrate and validate analytic waveform models, construct improved models of the dependence of remnant properties on the initial masses and spins of the black holes, and explore the dynamics of the strongly warped spacetime during the merger. In a companion paper, we use these methods to explore the extremality of apparent horizons in numerical simulations [50].

The remainder of this paper is organized as follows. We summarize our techniques in Sec. II, focusing on new improvements to our algorithm that enable us to simulate higher black-hole spins more robustly. We present three new simulations in Sec. III, and we present results in Sec. IV, including a comparison of the emitted gravitational waveforms with analytical predictions and also a comparison of the evolution of the black-hole masses and spins with analytic predictions. We briefly conclude in Sec. V.

\section{TECHNIQUES}

We carry out numerical simulations with the Spectral Einstein Code (SpEC) [47]. We construct [51] quasiequilibrium $[48,52]$ constraint-satisfying [53] initial data based on a weighted superposition of two boosted, spinning Kerr-Schild black holes [48]. We use an iterative method to produce initial data with low eccentricity [5456].

We use a generalized harmonic formulation [57-60] of Einstein's equations and damped harmonic gauge [61-63] to evolve the initial data. The adaptively-refined [36, 64] grid extends from pure-outflow excision boundaries just inside the apparent horizons [63, 65-67] to an artificial outer boundary, where we enforce constraint-preserving boundary conditions $[60,68,69]$. The grid has only one excision boundary after the holes merge [65, 66]. We use a pseudospectral fast-flow algorithm [70] to find apparent horizons, and we compute spins on these apparent horizons using the approximate Killing vector formalism of Cook, Whiting, and Owen [71, 72].

In the remainder of this section, we describe new techniques that allow simulations of binaries with large blackhole spins. Large spins are difficult for two reasons. First, the metric gradients near the black-hole horizons become larger with larger spin, making increased numerical resolution necessary in this region. Second, black-hole excision is more difficult: in SpEC, we remove the physical singularity inside each black hole by placing an artificial excision boundary just inside each apparent horizon 
and evolve only the region exterior to all excision boundaries. We find that the maximum required coordinate distance between an excision boundary and the corresponding horizon becomes smaller with larger spin, so that our algorithm for dynamically adjusting the excision boundaries to track the size, shape, and motion of the horizons must be more accurate. We consider both of these difficulties below.

Not all of the improvements discussed here were necessary for all of the simulations described in Sec. III. For example, the simulation discussed in Sec. III A succeeded without some of the grid and control system improvements; however, these improvements became necessary when simulating even larger black-hole spins (Sec. III B) or allowing generic spin directions and unequal masses (Sec. III C).

\section{A. Grid improvements}

Meeting the need for high resolution near the horizons is accomplished via spectral adaptive mesh refinement [64]. This includes both $p$-type refinement (changing the number of collocation points in a given spectral subdomain) and $h$-type refinement (adding, removing, or changing the distribution of subdomains). The simulations described here used the algorithm detailed in [64], with adjustments to default parameters so as to allow for higher resolution. In particular: We increased the number of radial collocation points in a spherical subdomain that forces $h$-refinement from 20 points to 40 , we disabled angular $h$-refinement in the spherical subdomains that touch the excision boundary so as to retain a single spherical subdomain at this boundary, and we increased the allowed number of spherical-harmonic coefficients in spherical shells from $L=40$ to $L=80$. Note that most of these changes (such as allowing up to $L=80$ ) were necessary only for a small portion of the simulation when the horizons are highly distorted, such as during the initial "junk radiation" transients (when spurious gravitational radiation is emitted as the $\mathrm{BBH}$ relaxes to equilibrium) and near the moment of merger.

We also reduced the initial distance between the apparent horizons and the excision boundaries. To understand this change, note that when solving elliptic equations for initial data, the excision boundaries are made to coincide exactly with the apparent horizons via boundary conditions imposed on those surfaces. But for the evolution, the excision boundaries must be slightly inside the horizons, so that the horizons are fully contained in the computational domain and therefore can be determined by the apparent horizon finder. To accomplish this, after the initial data have been determined, these data are extrapolated slightly inside the horizons to a new excision boundary, before the evolution begins. For large spins, this extrapolation occurs in the region where metric gradients are growing rapidly as $r$ decreases, so placing the excision boundary at a larger $r$ reduces those gradients.
To carry out some of the simulations shown here, we moved the initial excision boundary radius from $94 \%$ to $98 \%$ of the initial horizon radius.

\section{B. Control system improvements}

Several of the improvements necessary for handling high spins involve control systems used to adjust mappings between coordinate systems. These control systems and the mappings are described in detail in [66]. Here we briefly summarize important points, and we discuss key differences from [66].

\section{Summary of size and shape control systems}

In SpEC, we remove the physical singularity inside each black hole by placing an artificial excision boundary just inside each apparent horizon, evolving only the region exterior to all excision boundaries. We use multiple coordinate systems to handle excision of black holes that are moving and changing shape [63, 65, 66, 73-76]. We call "inertial coordinates" $\bar{x}^{i}$ those asymptotically inertial coordinates in which the black holes orbit each other, have a distorted and dynamical shape, and approach each other as energy is lost to gravitational radiation. We apply spectral methods in a different coordinate system, "grid coordinates" $x^{i}$, in which the excision boundaries are spherical and time-independent. We connect these two coordinate systems with an analytic mapping function $\mathcal{M}: x^{i} \rightarrow \bar{x}^{i}$ that depends on a set of time-dependent parameters $\lambda(t)$. These parameters $\lambda(t)$ are adjusted automatically by feedback control systems so that, as the apparent horizons of the black holes move and change shape (in the inertial frame), the excision boundaries are mapped to inertial-coordinate surfaces that follow this motion and remain just inside the apparent horizons.

The control of all parameters $\lambda(t)$ is accomplished in the same way, using a general control system we have developed, as described in [66]. The part of the algorithm that distinguishes one $\lambda(t)$ from another is the specification of the control error $Q(t)$, which is different for each control parameter. For example, the $\lambda_{\text {Scaling }}(t)$ that represents the distance between the excision boundaries has a different $Q(t)$ than the matrix $\lambda_{\text {Rotation }}(t)$ that represents the rotation of the inertial coordinates with respect to the grid coordinates. If there exists a desired value of $\lambda(t)$, call it $\lambda_{\text {target }}$, which depends on observables $A$ (such as the positions or shapes of the apparent horizons) but does not depend on $\lambda$ itself, then we define

$$
Q(t)=\lambda_{\text {target }}(A)-\lambda(t) .
$$

For more general situations in which $\lambda_{\text {target }}$ depends on $\lambda$ itself, we generalize the above definition: we require that $\lambda$ takes on its desired value when $Q=0$, and we require 
that

$$
\frac{\partial Q}{\partial \lambda}=-1+\mathcal{O}(Q)
$$

Given $Q(t)$, our algorithm adjusts the corresponding $\lambda(t)$ so that $Q(t)$ is driven towards zero; this driving occurs on a timescale $\tau_{d}$ that is determined dynamically and that is different for each control system.

The full map from grid to inertial coordinates is $\bar{x}^{i}=$ $\mathcal{M} x^{i}$, where

$$
\begin{aligned}
\mathcal{M}= & \mathcal{M}_{\text {Translation }} \circ \mathcal{M}_{\text {Rotation }} \circ \mathcal{M}_{\text {Scaling }} \\
& \circ \mathcal{M}_{\text {Skew }} \circ \mathcal{M}_{\text {CutX }} \circ \mathcal{M}_{\text {Shape }}
\end{aligned}
$$

Each of these maps is described in detail in Sec. 4 of [66].

Shape control. Here we are concerned only with the last map, $\mathcal{M}_{\text {Shape }}$, which is defined as:

$x^{i} \mapsto x^{i}\left(1-\sum_{H} \frac{f_{H}\left(r_{H}, \theta_{H}, \phi_{H}\right)}{r_{H}} \sum_{\ell m} Y_{\ell m}\left(\theta_{H}, \phi_{H}\right) \lambda_{\ell m}^{H}(t)\right)$.

The index $H$ goes over each of the two excised regions $A$ and $B$, and the map is applied to the grid-frame coordinates. The polar coordinates $\left(r_{H}, \theta_{H}, \phi_{H}\right)$ centered about excised region $H$ are defined in the usual way, the quantities $Y_{\ell m}\left(\theta_{H}, \phi_{H}\right)$ are spherical harmonics, and $\lambda_{\ell m}^{H}(t)$ are expansion coefficients that parameterize the map near excision region $H$; these $\lambda_{\ell m}^{H}(t)$ are the coefficients that we adjust using a control system. The function $f_{H}\left(r_{H}, \theta_{H}, \phi_{H}\right)$ is chosen to be unity near excision region $H$ and zero near the other excision region, so that the distortion maps for the two black holes are decoupled; see Eq. 72 and Fig. 4 of [66] for a precise definition of $f_{H}\left(r_{H}, \theta_{H}, \phi_{H}\right)$. In the following, the control systems for each excised region $H$, while independent, are identical in operation, so we will omit the $H$ labels for simplicity.

We control $\lambda_{\ell m}(t)$ so that each excision boundary is driven to the same shape as the corresponding apparent horizon; this results in conditions on $\lambda_{\ell m}(t)$ for $\ell>0$, but leaves $\lambda_{00}(t)$ unconstrained [66].

Size control. The size of the excision boundary, as encoded in the remaining coefficient $\lambda_{00}(t)$, must satisfy two conditions.

Horizon tracking. The first is that the excision boundary remains inside the apparent horizon. To satisfy this condition, we first write the shape of each apparent horizon as an expansion in spherical harmonics, parameterized in terms of polar coordinates about the center of the corresponding excision boundary,

$$
\hat{r}^{\mathrm{AH}}(\hat{\theta}, \hat{\phi})=\sum_{\ell m} \hat{S}_{\ell m} Y_{\ell m}(\hat{\theta}, \hat{\phi}),
$$

where the intermediate frame $\hat{x}^{i}$ is connected to the grid frame by the map

$$
\mathcal{M}_{\text {Distortion }}=\mathcal{M}_{\text {CutX }} \circ \mathcal{M}_{\text {Shape }} .
$$

By construction, $\mathcal{M}_{\text {Distortion }}$ leaves invariant the centers of the excision boundaries, and the angles with respect to these centers. Then we choose

$$
Q=\dot{\hat{S}}_{00}(\Delta r-1)-\dot{\lambda}_{00}
$$

where

$$
\Delta r=1-\frac{\left\langle\hat{r}_{\mathrm{EB}}\right\rangle}{\left\langle\hat{r}_{\mathrm{AH}}\right\rangle}
$$

is the relative difference between the average radius of the apparent horizon (in the intermediate frame) and the average radius of the excision boundary. The angle brackets in Eq. (8) represent averaging over angles. Choosing $Q(t)$ according to Eq. (7) drives $d / d t(\Delta r)$ towards zero, so that the excision boundary remains a fixed (relative) distance inside the apparent horizon.

Characteristic speed tracking. The second condition that must be satisfied by $\lambda_{00}(t)$ involves characteristic speeds of the evolved Einstein equations: well-posedness of our system of equations requires that all of the characteristic speeds be non-negative, i.e. characteristic fields must flow into the black hole. The minimum characteristic speed at each excision boundary is given by

$$
v=-\alpha-\bar{\beta}^{i} \bar{n}_{i}-\bar{n}_{i} \frac{\partial \bar{x}^{i}}{\partial t},
$$

where $\alpha$ is the lapse, $\bar{\beta}^{i}$ is the shift, and $\bar{n}_{i}$ is the normal to the excision boundary pointing out of the computational domain, i.e., toward the center of the hole. It is possible to write [66]

$$
v=v_{0}+\hat{n}_{i} \frac{x^{i}}{r} Y_{00} \dot{\lambda}_{00},
$$

where $v_{0}$ collects all terms that are independent of $\dot{\lambda}_{00}$. Therefore, a control system that controls $\dot{\lambda}_{00}$ and drives $v$ to some target value $v_{T}$ can be constructed by defining the control error

$$
Q=\left(\min (v)-v_{T}\right) /\langle-\Xi\rangle,
$$

where

$$
\Xi=\hat{n}_{i} \frac{x^{i}}{r} Y_{00},
$$

and the minimum is over the excision boundary. Note that $\Xi<0$ because $\hat{n}_{i}$ and $x^{i} / r$ point in opposite directions.

Switching between horizon and characteristic speed tracking. Note that Eqs. (7) and (11) specify two different control systems that control the same degree of freedom, $\lambda_{00}$ : the first control system, which we call "horizon tracking", adjusts $\dot{\lambda}_{00}$ to control $\Delta r$, and the other, which we call "characteristic speed tracking", adjusts $\dot{\lambda}_{00}$ to control $v$. Both $\Delta r$ and $v$ must remain nonnegative for a successful evolution, but we cannot use both Eqs. (7) 
and (11) simultaneously. Furthermore, changes in $\dot{\lambda}_{00}$ affect $\Delta r$ and $v$ in the opposite direction: if $\dot{\lambda}_{00}$ increases, $\Delta r$ increases, but the characteristic speed $v$ decreases.

In practice (Sec. II B 3), we now alternate between the two control systems, Eqs. (7) and (11). We monitor both $v$ and $\Delta r$ as functions of time and predict whether either of these quantities is likely to become negative in the immediate future; if so, we estimate the timescale $\tau_{v}$ or $\tau_{\Delta r}$ on which this will occur. If $\tau_{\Delta r}$ is small enough, i.e. $\Delta r$ is in imminent danger of becoming negative, we use Eq. (7) to control $\Delta r$. If $\tau_{v}$ is small enough that $v$ is in danger of becoming negative, we use Eq. (11) to control $v$. The details of how we make these decisions have been improved since the description in Sec. 5.3 of [66], so we describe the improved algorithm below.

\section{Improvements in gain scheduling}

We now describe improvements in the control systems that were necessary for our new high-spin simulations to succeed.

Comoving characteristic speed as a control system diagnostic. We define a new quantity $v_{c}$ which we call the comoving characteristic speed:

$$
\begin{aligned}
v_{c}= & -\alpha-\bar{\beta}^{i} \bar{n}_{i}-\bar{n}_{i} \frac{\partial \bar{x}^{i}}{\partial \hat{t}} \\
& +\hat{n}_{i} \frac{x^{i}}{r}\left[Y_{00} \dot{\hat{S}}_{00}(\Delta r-1)+\sum_{\ell>0} Y_{\ell m}(\theta, \phi) \dot{\lambda}_{\ell m}(t)\right] .
\end{aligned}
$$

The comoving characteristic speed $v_{c}$ is what the characteristic speed $v$ would be if $Q(t)$ in Eq. (7) were exactly zero, i.e. if $\Delta r$ were constant in time. In other words, if we turn on horizon tracking, the control system drives $v$ toward $v_{c}$. This tells us (for instance) that if we find $v_{c}<0$, we should not use horizon tracking, since horizon tracking would drive $v$ to a negative value. The instantaneous value of $v_{c}$ is independent of $\dot{\lambda}_{00}$ and roughly independent of $\lambda_{00}$; the only dependence on $\lambda_{00}$ comes from the smooth spatial variation of the metric functions. Hence, $v_{c}$ is a useful quantity for separating the effects of the control system for $\dot{\lambda}_{00}$ from the effects of other control systems.

One way we use $v_{c}$ is in determining whether our control system for $\dot{\lambda}_{00}$ will fail. During a simulation, $v_{c}$ is usually positive, but it routinely becomes negative for short periods of time, particularly when the shapes of the horizons are changing rapidly, for example near $t=0$ when the black holes are ringing down from initial "junk radiation" transients. However, if $v_{c}$ becomes negative and remains so indefinitely, our control system for $\dot{\lambda}_{00}$ must eventually fail. This is because for $v>0$ and $v_{c}<0$, $\Delta r$ must be decreasing, so if we keep $v>0$ the excision boundary will eventually intersect the apparent horizon.

Control error damping timescale improvements. For many of the high-spin SpEC simulations that failed before we made the improvements described in this paper, we observed that $v_{c}<0$ for an extended period of time. This was caused by inaccurate control systems for the $\lambda(t)$ parameters other than $\lambda_{00}$; in particular, the shape parameters $\lambda_{\ell m}$ for $\ell>0$. In other words, the shape and position of the excision boundary differed from the shape and position of the horizon by a sufficient amount that it was not possible to make both $v>0$ and $\Delta r>0$ everywhere by adjusting only the radial motion of the excision boundary, $\lambda_{00}$.

This particular problem was fixed by changing the algorithm for setting the tolerance on the control error $Q(t)$, for all $Q(t)$ except $Q_{00}$. Associated with each of our control systems is a timescale parameter $\tau_{d}$ which is adjusted dynamically. The control error $Q(t)$ is damped like $e^{-t / \tau_{d}}$, under the assumption that $\tau_{d}$ is smaller than all other timescales in the problem. Therefore decreasing $\tau_{d}$ results in smaller values of $Q(t)$. The previous method of adjusting $\tau_{d}$ is described in Sec. 3.3 of [66]: at regular time intervals $t_{i}$, the timescale is changed according to

$$
\tau_{d}^{i+1}=\beta \tau_{d}^{i}
$$

where

$\beta= \begin{cases}0.99, & \text { if } \dot{Q} / Q>-1 / 2 \tau_{d} \text { and }|Q| \text { or }\left|\dot{Q} \tau_{d}\right|>Q_{t}^{\operatorname{Max}} \\ 1.01, & \text { if }|Q|<Q_{t}^{\mathrm{Min}} \text { and }\left|\dot{Q} \tau_{d}\right|<Q_{t}^{\mathrm{Min}} \\ 1, & \text { otherwise. }\end{cases}$

Here $Q_{t}^{\mathrm{Min}}$ and $Q_{t}^{\mathrm{Max}}$ are thresholds for the control error $Q$, set to constant values

$$
\begin{aligned}
Q_{t}^{\mathrm{Max}} & =\frac{2 \times 10^{-3}}{m_{A} / m_{B}+m_{B} / m_{A}} \\
Q_{t}^{\mathrm{Min}} & =\frac{1}{4} Q_{t}^{\mathrm{Max}} .
\end{aligned}
$$

In the new algorithm, we make three changes. The first is that $Q_{t}^{\mathrm{Max}}$ is no longer a constant: instead, it is chosen to be $Q_{t}^{\text {Max }}=a \Delta r_{\min }$, where $a$ is a constant (typically chosen to be $0.05\left(m_{A}+m_{B}\right)$ for those $Q$ values with dimensions of length, and 0.05 for those $Q$ values that are dimensionless) and $\Delta r_{\text {min }}$ is the minimum relative distance between the excision boundary and the apparent horizon:

$$
\Delta r_{\min }=\min _{\hat{\theta}, \hat{\phi}}\left(1-\frac{\hat{r}_{\mathrm{EB}}(\hat{\theta}, \hat{\phi})}{\hat{r}_{\mathrm{AH}}(\hat{\theta}, \hat{\phi})}\right) .
$$

The second change is that we define an estimate of the time that the horizon will cross the excision surface

$$
\tau_{\Delta r \text { cross }}=-\Delta r_{\min }\left(\frac{d}{d t} \Delta r_{\min }\right)^{-1}
$$

and if $\tau_{\Delta r \text { cross }}>0$ and $\tau_{d}^{i}>\tau_{\Delta r \text { cross }}$, then we set $\tau_{d}^{i+1}=$ $\tau_{\Delta r \text { cross }}$ instead of using Eq. (14).

Both of the above changes force each $Q(t)$ to be closer to zero when the excision boundary approaches the horizon. A third, minor, change we make in the algorithm 


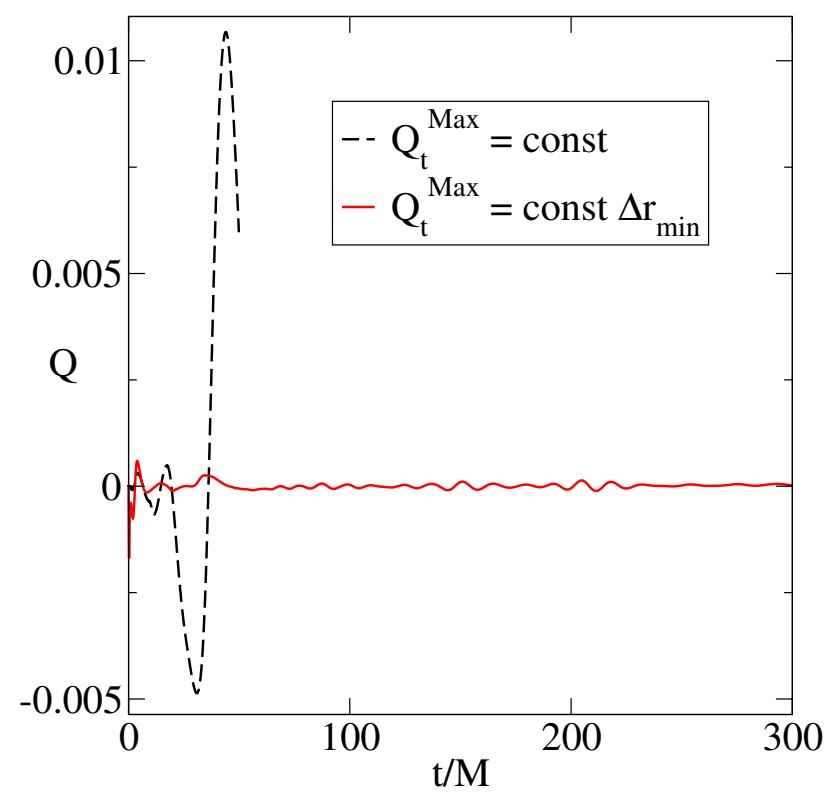

FIG. 1. The control error $Q(t)$ associated with one particular control system for two different $S_{0.994}^{++}$simulations which differ only in the treatment of $Q_{t}^{\mathrm{Max}}$ for that control system. The black dashed curve shows the case in which $Q_{t}^{\mathrm{Max}}$ is a constant, given by Eq. (16), and the red solid curve shows the case in which $Q_{t}^{\mathrm{Max}}$ is chosen to be $0.05 \Delta r_{\min }$, with $\Delta r_{\min }$ given by Eq. (18). The former simulation crashes at $t \sim 50 M$.

affects only the behavior of $Q(t)$ at early times: the initial values of each $\tau_{d}$ were decreased so that each $Q(t)$ is smaller at earlier times; these initial values are specified separately from the tolerances $Q_{t}^{\mathrm{Max}}$ that determine when $\tau_{d}$ is modified. The effect of the first change, setting $Q_{t}^{\operatorname{Max}}$ proportional to $\Delta r_{\min }$, is illustrated in Fig. 1 for one particular control system. ${ }^{1}$ In Fig. 1 and the remainder of the paper, $M \equiv m_{A}+m_{B}$ is the sum of the Christodoulou masses of the two black holes at the time $t_{\text {relax }}$ when the initial "junk radiation" transients have decayed away.

\section{Size control: switching between Eqs. (7) and (11).}

At every time step, the control system for $\lambda_{00}$ is governed by a $Q$ given by either Eq. (7) or Eq. (11), with an associated damping timescale $\tau_{d}$ and (if using characteristic speed control) a target speed $v_{T}$. At regular intervals (typically every time step), the algorithm has an opportunity to change from using Eq. (7) to using Eq. (11)

1 The $Q(t)$ illustrated here is the one for the control system that computes a smooth approximation $\hat{r}_{\mathrm{AH}}^{a p p x}(t)$ to the average horizon radius; this approximate value is used to compute $\dot{\hat{S}}_{00}$ and $\Delta r$ in Eq. (7), in order to reduce the number of calls to the computationally expensive horizon finder (see section 7 and Eq. (108) of [66] for details). or vice versa, and to choose a new value of $\tau_{d}$ and (if using characteristic speed control) $v_{T}$. Here we describe how we make these choices. A previous version of this algorithm was described in [66], but many improvements have been made since then.

Because the goal of the $\lambda_{00}$ control system is to keep both $v$ and $\Delta r_{\min }$ positive, we regularly monitor $v$ and $\Delta r_{\min }$ as functions of time. We predict whether either of these quantities is likely to become negative in the immediate future, and if so, we estimate the timescale $\tau_{v}$ or $\tau_{\Delta r_{\min }}$ on which this will occur, using the method described in Appendix $\mathrm{C}$ of [66]. Because the sign of $v_{c}$ is important to the success of horizon tracking, we also monitor $v_{c}$ as a function of time, and if it is positive and decreasing, we predict the timescale $\tau_{v_{c}}$ on which it will become negative. If $v, v_{c}$, or $\Delta r_{\text {min }}$ are increasing instead of decreasing, we define the corresponding timescale $\tau_{v}$, $\tau_{v_{c}}$, or $\tau_{\Delta r_{\min }}$ to be infinite.

We begin by determining whether $v$ is in imminent danger of becoming negative, so that some immediate action must be taken to prevent this from occurring. We regard $v$ to be in danger if $\tau_{v}<\tau_{d}$ and $\tau_{v}<\tau_{\Delta r_{\min }}$. Furthermore, if characteristic speed control is in effect, we additionally require $\tau_{v}<\sigma_{3} \tau_{d}$ and $v<\sigma_{4} v_{T}$ to deem $v$ in danger; here $\sigma_{3} \lesssim 1$ and $\sigma_{4} \sim 1$ are constants, typically chosen to be 0.99 and 1.1 , respectively ${ }^{2}$. The first requirement, $\tau_{v}<\sigma_{3} \tau_{d}$, prevents the algorithm from switching back and forth between characteristic speed control and horizon tracking on each time step. The second requirement, $v<\sigma_{4} v_{T}$, prevents the control system from rapidly decreasing the characteristic speed to achieve a target $v_{T}$ that is less than $v$.

If $v$ is deemed to be in danger, the action taken to prevent $v$ from becoming negative depends on the current state of the control system. If characteristic speed control is in effect, then it remains in effect, and $\tau_{d}$ is set equal to $\tau_{v}$ in order to drive $v$ towards $v_{T}$ more quickly. If horizon tracking is in effect, and if $v_{c}<0$ or $v_{c}$ is decreasing, then characteristic speed control goes into effect, with $v_{T}=$ $\sigma_{5} v$, and $\tau_{d}$ is left unchanged. The constant $\sigma_{5}$, typically 1.01, prevents the control system from switching back and forth on each timestep. Finally, if horizon tracking is in effect, and if $v_{c}>0$ and $v_{c}$ is nondecreasing, then horizon tracking remains in effect and we reduce $\tau_{d}$ by a factor of $\sigma_{6}<1$ (typically 0.99). This change is all that is required because horizon tracking will drive $v$ toward $v_{c}$, which is in no danger of becoming negative.

If $v$ is deemed not to be in danger, then we check whether $\Delta r_{\min }$ is in danger of soon becoming negative. We regard $\Delta r_{\min }$ to be in danger if $\tau_{\Delta r_{\min }}<\tau_{v}$ and if $\tau_{\Delta r_{\min }}<\sigma_{1} \tau_{d}$, where $\sigma_{1}$ is a constant typically chosen to be 20. Furthermore, if horizon tracking is in effect, we additionally require $\tau_{\Delta r_{\min }}<\sigma_{7} \tau_{d}$ to deem $\tau_{\Delta r_{\min }}$ in danger, where $\sigma_{7}<1$ is usually chosen to be 0.99 ; this

\footnotetext{
${ }^{2}$ Labels for control system constants like $\sigma_{i}$ and $\eta$ are consistent with the notation in Ref. [66].
} 


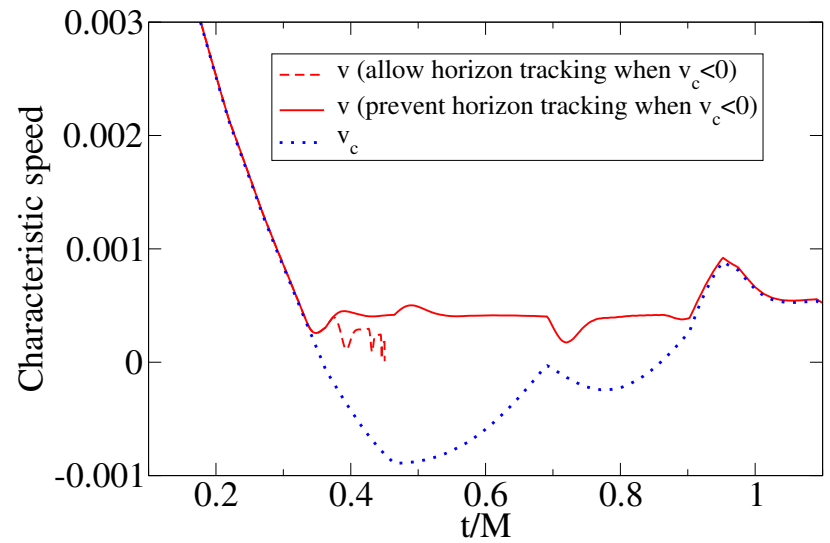

FIG. 2. Characteristic speed $v$ and comoving characteristic speed $v_{c}$ for two different $S_{0.994}^{++}$simulations that differ only in the algorithm for treating the situation in which $\Delta r_{\text {min }}$ is deemed in danger while characteristic speed control is in effect and $v_{c}<0$. The dashed red curve shows $v$ for a simulation in which horizon tracking becomes active in this situation; the code crashes early, at only $t \sim 0.4 M$. The solid red curve shows $v$ for a simulation in which for this situation characteristic speed control remains in effect, but the target characteristic speed is reduced as described in the text. The quantity $v_{c}$ is the same for both simulations.

condition prevents the control system from switching on every time step.

If $\Delta r_{\min }$ is in danger, the action again depends on the state of the control system and other variables. If horizon tracking is in effect, then it remains in effect, and $\tau_{d}$ is set equal to $\tau_{\Delta r_{\min }}$ in order to drive $\Delta r_{\min }$ to a constant more quickly. If characteristic speed control is in effect and if $v_{c}>0$, then horizon tracking goes into effect, and $\tau_{d}$ is set equal to $\tau_{\Delta r_{\min }}$. We require $v_{c}>0$ to activate horizon tracking because horizon tracking drives $v$ towards $v_{c}$, and we wish to maintain $v>0$; if horizon tracking becomes active even if $v_{c}<0$, the simulation often fails, as shown in Fig. 2. To solve the problem illustrated by Fig. 2, when the code finds that $\Delta r_{\min }$ is in danger while characteristic speed control is in effect and if $v_{c}<0$, then the code allows characteristic speed control to remain in effect, but it sets the new $\tau_{d}$ to $\min \left(\tau_{d}, \tau_{\Delta r_{\min }}\right)$, and it reduces $v_{T}$ to $\eta v$, where $\eta<1$ is a constant typically chosen to be 0.125 . Reducing the target $v_{T}$ will reduce $v$ but will increase $\Delta r_{\min }$. If $v_{c}<0$ for an extended period of time, several such reductions of $v_{T}$ will occur as needed. As mentioned above, if $v_{c}<0$ and remains so, this algorithm must eventually fail; the way to prevent such a failure is to adjust the control systems other than the one for $\lambda_{00}$ to attempt to make $v_{c}$ positive, as discussed in Sec. II B 2.

If neither $v$ nor $\Delta r_{\text {min }}$ are in imminent danger of becoming negative, then the system attempts to find an equilibrium using horizon tracking. If characteristic speed control is in effect, and if $v_{c}>0, v_{c} \geq 0$, and either $v>v_{T}$ or $v_{c}>v$, then horizon tracking goes into effect, using the current $\tau_{d}$. However, if both $v$ and $v_{c}$ are de- creasing, horizon tracking does not go into effect unless $v$ is decreasing faster than $v_{c}$ and $\tau_{v_{c}}>\sigma_{2} \tau_{d}$, where $\sigma_{2}$ is a constant we usually set to 5 . The purpose of these various conditions on $v, v_{c}$, and their derivatives and predicted zero-crossing times is to prevent horizon tracking from going into effect when it is likely that a switch back to characteristic speed control will soon be necessary. For example, if $\dot{v}_{c}<0$ and $v_{c}$ is decreasing faster than $v$, then we anticipate that $v_{c}$ will soon become negative, in which case horizon tracking is inappropriate because it would drive $v$ towards zero.

The behavior of the control system depends on various constants $\sigma_{i}(1<i<7)$ and $\eta$ described above; these constants govern decisions made by the algorithm. These constants have restricted values (e.g. $\eta$ should not be greater than unity), but they were chosen without any fine tuning. Changing their values slightly will change details such as the exact value of $\tau_{d}$ at a particular timestep, but we expect that small changes in parameters will not change whether a simulation succeeds or fails, and will change physical results only at the level of truncation error (because the control system changes the grid coordinates).

Occasionally when horizon tracking is in effect, we find that the value of $\Delta r_{\min }$ is excessively large or small. If it is excessively small, then $\tau_{d}$ becomes small, and we are forced to reduce the timestep in the evolution equations to keep the control system stable, resulting in a large computational expense. If it is too large, then the excision boundary lies deep inside the horizon, and excessive computational resources are needed to resolve the large gradients. Therefore, we allow a drift term to sometimes be added to Eq. (7), as discussed in [66].

\section{SIMULATIONS}

We present three new simulations, summarized in Table I. We will refer to quantities defined in Table I throughout the remainder of this paper. The techniques described in Sec. II were essential to the successful completion of these simulations.

\section{A. Equal-mass, aligned spins $\chi=0.99$}

The first simulation we present, and refer to as $S_{0.99}^{++}$, is an equal-mass case in which each black hole has a spin of $\chi=0.99$ aligned with the orbital angular momentum. At $t=t_{\text {relax }}$ the simulation has $M \omega_{\text {orb }}=0.0154$, where $M$ is the sum of the relaxed Christodoulou masses. The binary then evolves through 25 orbits, merger and ringdown. This simulation took 83 days on 48 cores for the highest resolution.

To assess numerical convergence, we perform several simulations that are identical except for the numerical resolution, which we label by an integer $N$. Larger $N$ corresponds to finer resolution, but the absolute scale 


\begin{tabular}{lc|ccccccccccc|rc|cc} 
Name & Catalog ID & $t_{\text {relax }}$ & $q^{r}$ & $m_{A}^{r}$ & $m_{B}^{r}$ & $M \omega_{\text {orb }}^{r}$ & $\chi_{A}^{r}$ & $\theta_{A}^{r} / \pi$ & $\phi_{A}^{r} / \pi$ & $\chi_{B}^{r}$ & $\theta_{B}^{r} / \pi$ & $\phi_{B}^{r} / \pi$ & $10^{4} e$ & $N$ & $M_{f}$ & $\chi_{f}$ \\
\hline \hline$S_{0.99}^{++}$ & SXS:BBH:0177 & 320.0 & 1.0 & 0.5 & 0.5 & 0.0154 & 0.989 & 0.00 & - & 0.989 & 0.00 & - & 12.6 & 25.4 & 0.888 & 0.949 \\
$S_{0.994}^{++}$ & SXS:BBH:0178 & 640.0 & 1.0 & 0.5 & 0.5 & 0.0157 & 0.994 & 0.00 & - & 0.994 & 0.00 & - & 8.6 & 25.4 & 0.887 & 0.950 \\
$S_{0.20}^{0.99}$ & SXS:BBH:0179 & 380.0 & 1.5 & 0.6 & 0.4 & 0.0148 & 0.991 & 0.00 & 0.73 & 0.200 & 0.24 & 0.23 & 322.4 & 23.8 & 0.922 & 0.897
\end{tabular}

TABLE I. Summary of physical simulation parameters. Data are publicly available online [77] indexed by their Catalog ID. Quantities with an $r$ superscript are reported at time $t=t_{\text {relax }}$, the time after the initial "junk radiation" transients have settled down: $q$ is the mass ratio, $m_{H}$ is the Christodoulou mass of an individual black hole (where $H$ represents black hole $A$ or $B), M \omega_{\text {orb }}$ is the orbital frequency, $\chi_{H}$ is the dimensionless spin, $\theta_{H}$ is the angle between $\vec{\omega}_{\text {orb }}$ and $\vec{\chi}_{H}$, and $\phi_{H}$ is the angle between the separation vector and the component of $\vec{\chi}_{H}$ in the orbital plane. The remaining quantities are eccentricity $e$, number of orbits $N$ from $t=0$ to merger, final Christodoulou mass $M_{f}$, and final spin magnitude $\chi_{f}$.

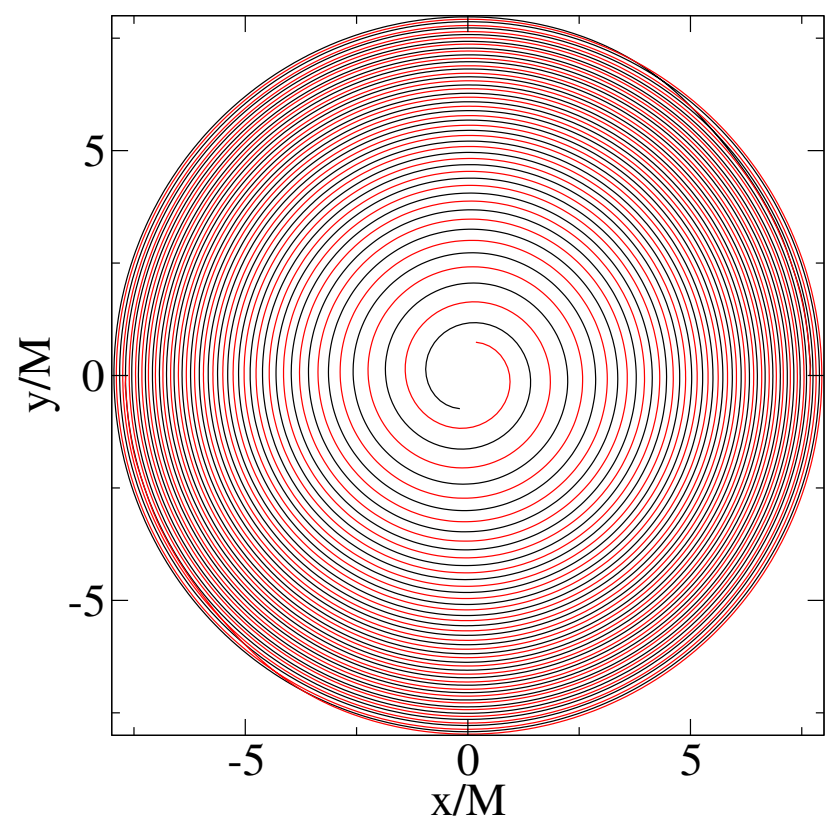

FIG. 3. The trajectories of the centers of the individual apparent horizons for the highest resolution of $S_{0.99}^{++}$.

of $N$ is different for different physically distinct simulations. The value of $N$ enters the simulation through the tolerance in adaptive mesh refinement (AMR): the AMR truncation error tolerance is chosen to be proportional to $e^{-N}$. For each value of $N$, we compute the complex phase $\phi$ of the $\ell=2, m=2$ component of $\Psi_{4}$. We then take the difference $\Delta \phi$ between $\phi$ computed using otherwise-identical simulations using different values of $N$.

Figure 4 shows these differences for $S_{0.99}^{++}$. No alignment of the waveforms in time or phase has been performed. Note the rapid convergence: $\Delta \phi$ between $N=3$ and $N=4$ (labeled " $4-3$ ") is significantly smaller than $\Delta \phi$ between the two lower resolutions. Also note that the difference " $3-2$ " is nearly the same as " $4-2$ ", indicating that this difference effectively measures the numerical truncation error in the $N=2$ simulation. Similarly, the difference " $4-3$ " represents the numerical truncation error in the $N=3$ simulation. Furthermore, one would expect that the truncation error in the $N=4$ simulation

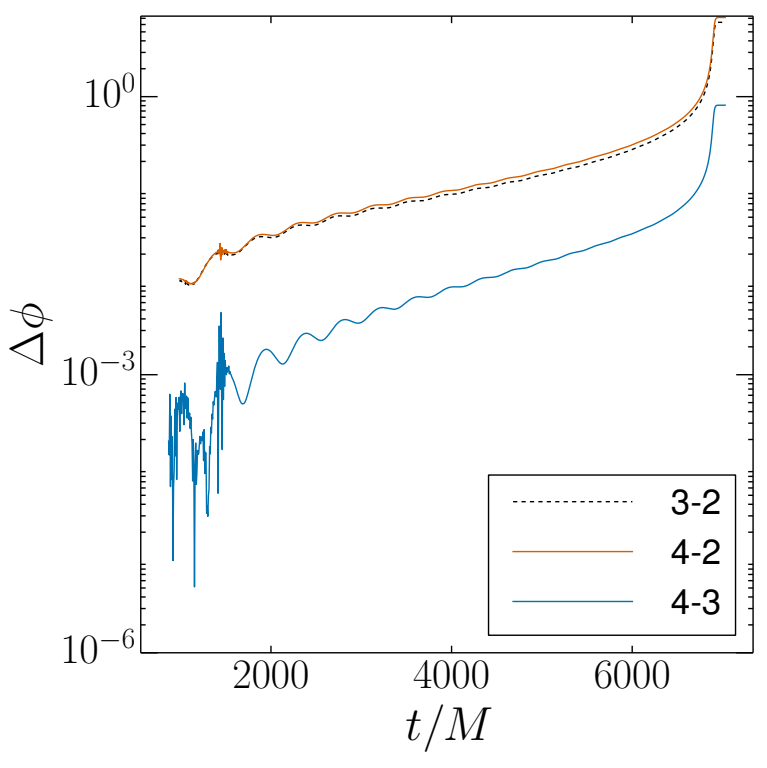

FIG. 4. Convergence test for $S_{0.99}^{++}$. Shown are gravitationalwave phase differences between $\Psi_{4}$ computed using different values of the numerical resolution parameter $N$. Several differences are shown, and labeled by the values of $N$ that are compared, e.g. " $3-2$ " means $N=3$ versus $N=2$. Waveforms are extracted at a finite radius $r=465 \mathrm{M}$, and no alignment of waveforms was performed.

is smaller than the " $4-3$ " curve by another order of magnitude (although it would be necessary to run an $N=5$ simulation to actually measure this).

In the $S_{0.99}^{++}$initial data, the spin of each black hole is 0.99 . When the system is evolved, the spins decrease very slightly for the first $\sim 10 M$ as initial transients propagate away from the horizons, as shown in the upper inset of Fig. 5. Then the spins level off and become roughly constant, but with a small negative slope. All values of resolution $N$ agree quite well, and the higher two resolutions are indistinguishable in Fig. 5. The spins decrease more rapidly just before merger $(t \sim 6400 M)$. The common horizon first appears with a spin greater than the final value, and then relaxes as the remnant black hole settles down, as shown in the lower inset of Fig. 5. The final spin is $\chi_{f}=0.948927(3)$, where the uncertainty is 


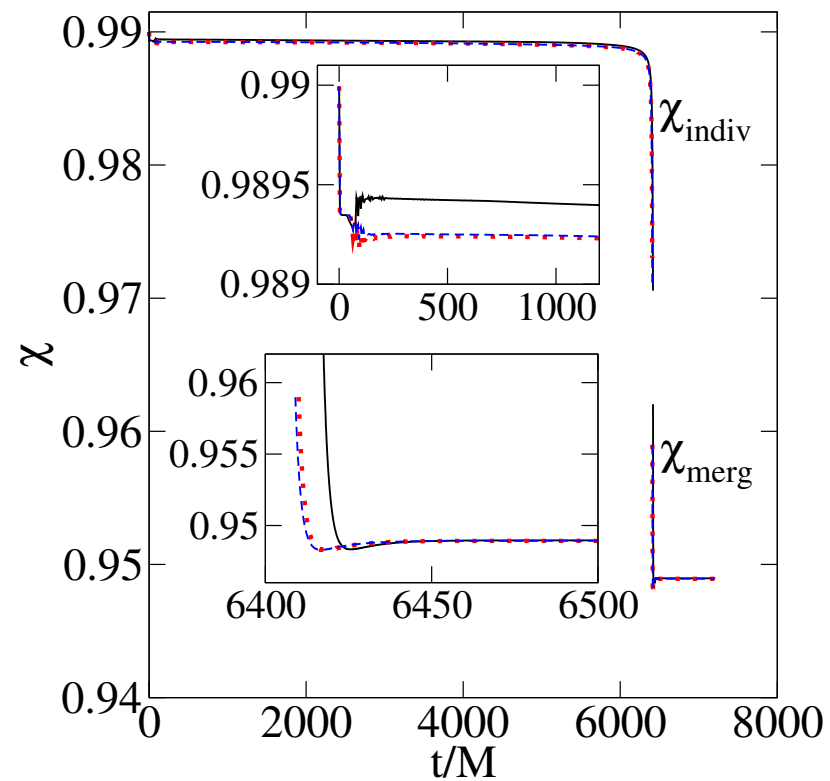

FIG. 5. Spin magnitude as a function of time for $S_{0.99}^{++}$. At early times, the spin of one of the apparent horizons is shown at resolutions $N=2$ (black solid), $N=3$ (red dotted) and $N=4$ (blue dashed). A closeup of early times is shown in the upper inset. At late times, the spin of the merged apparent horizon is shown as a function of time for the same resolutions, and a closeup of late times is shown in the lower inset.

the difference between the two highest resolution simulations.

The radiated energy fraction $E_{\text {rad }}$ is the relative change in energy of the binary from $t=-\infty$ to $t=\infty$ and can be computed from

$$
E_{\mathrm{rad}} \equiv 1-\frac{E_{\infty}}{E_{-\infty}}=1-\frac{M_{f}}{M} .
$$

The final Christodoulou mass $M_{f}$ is the energy of the system at $t=\infty$, because the remnant is in equilibrium at the end of the simulation; the total Christodoulou mass $M$ at $t=t_{\text {relax }}$ is the energy of the system at $t=-\infty$, because the individual black-hole masses change by less than one part in $10^{6}$ between $t=-\infty$ and $t=t_{\text {relax }}$ (see, e.g. Eq. 14 in Ref. [78]). We find that $E_{\mathrm{rad}}=11.26593(3) \%$, where the uncertainty is again the difference between the two highest resolutions.

The formulas from Ref. [41] predict $\chi_{f}=0.94933(8)$ and a radiated energy fraction $E_{\mathrm{rad}}=11.24(2) \%$, in good agreement with the simulations. While the fractional differences between the measured and predicted values are small, their uncertainty intervals are disjoint, i.e. our measurements lie outside the uncertainty interval of the formulas. This is because the error estimates in Ref. [41] did not account for the observed correlated trends in the fit residuals (as seen in the lower panels of Figs. 6 and 8 of Ref. [41]). As a result, extrapolating these formulas to initial spins above $\chi=0.97$ is expected to overestimate the final spin (see Fig. 6 in Ref. [41]) and underestimate

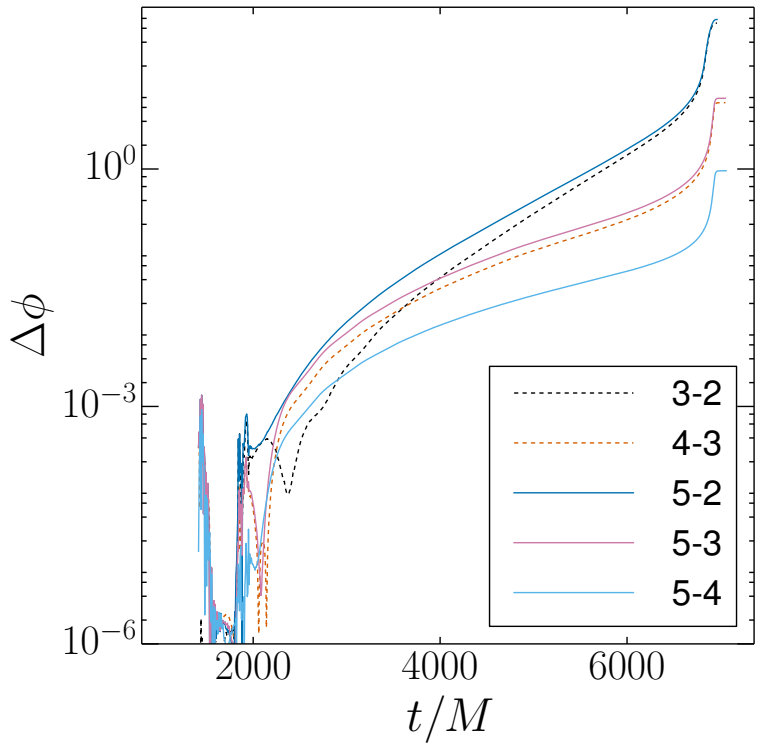

FIG. 6. Convergence test for $S_{0.994}^{++}$. Labels are the same as for Fig. 4. For $N \neq 5$, the simulations were started at $t_{\text {branch }}=1414 M$, using the $N=5$ solution as initial data.

the final radiated energy (see Fig. 8 in Ref. [41]), and this is what we find with $S_{0.99}^{++}$.

\section{B. Equal-mass, aligned spins $\chi=0.994$}

We repeated the equal-mass aligned-spin simulation above, but with a larger spin. We refer to this case as $S_{0.994}^{++}$. The initial data were chosen with $\chi=0.995$ for each black hole, but the spins drop to $\chi=0.9942$ after about $t=10 \mathrm{M}$ of evolution time, a much smaller timescale than the relaxation time $t_{\text {relax }}$ (this rapid initial decrease in spin can also be seen for $S_{0.99}^{++}$in the upper inset of Fig. 5). The simulation $S_{0.994}^{++}$represents the largest spin ever simulated for a black-hole binary. It has $M \omega_{\text {orb }}=0.0157$ at $t=t_{\text {relax }}$, and then proceeds through 25 orbits, merger, and ringdown. The highest resolution completed in approximately 71 days on 48 cores. Note that this simulation, $S_{0.994}^{++}$, was computationally cheaper than the lower-spin simulation, $S_{0.99}^{++}$, and achieved a smaller overall phase error (see Figs. 4 and 6). This is due to code optimization that was done between the time that the $S_{0.99}^{++}$and $S_{0.994}^{++}$simulations were carried out; for the same version of SpEC, there is actually a steep increase in computational cost as a function of spin.

Obtaining convergence was more difficult for this simulation than for $S_{0.99}^{++}$. The reason is that it is difficult to fully resolve the initial transients, sometimes called "junk radiation", that result from imperfect initial data. If these transients are unresolved, then the small changes in masses, spins, and trajectories caused by these tran- 
sients are effectively random, and therefore otherwiseidentical simulations run with different values of resolution $N$ will differ by random small amounts that will not converge with increasing $N$. So to investigate convergence, we remove the initial transients in the following way. We first carry out a simulation with one value of $N$, call it $N_{\text {base. }}$ In the case of $S_{0.994}^{++}, N_{\text {base }}$ represents the highest resolution. Then we choose some fiducial time $t=t_{\text {branch }}>t_{\text {relax }}$ at which we decide that the transients have decayed away. We then carry out simulations with $N \neq N_{\text {base }}$ starting at $t=t_{\text {branch }}$, using the $N=N_{\text {base }}$ solution as initial data. This procedure removes the effects of the transients from our convergence tests.

However, this procedure alone was insufficient to achieve convergence. When convergence is rapid enough in a particular subdomain so that adding a single grid point results in a large decrease in truncation error, it is possible for two different AMR truncation error tolerances, e.g. $e^{N}$ and $e^{N-1}$, to result in the same number of grid points for that subdomain. This makes the truncation error in that subdomain identical for two different values of $N$, which spoils convergence tests for simulations with those values of $N$. To remedy this problem in such cases, we increase the spacing in truncation error tolerance as a function of level $N$ : the truncation error tolerance is proportional to $10^{N}$ instead of $e^{N}$. This, combined with the procedure to remove the effect of transients, results in good convergence, as shown in Fig. 6.

The spin of the remnant black hole is $\chi_{f}=0.949931(5)$ and the radiated energy fraction is $E_{\mathrm{rad}}=11.351(5) \%$. The formulas in Ref. [41] predict $\chi_{f}=0.95021(8)$ and $E_{\mathrm{rad}}=11.30(2) \%$, in good agreement with the simulations. However, the uncertainty intervals of the measured and predicted values are disjoint for the same reason as explained in Sec. III A.

\section{Unequal-mass, precessing}

The final simulation we present is an unequal-mass case with $q=1.5$, in which the larger black hole has a spin of $\chi=0.99$ aligned with the orbital angular momentum, while the smaller black hole has a spin magnitude of $\chi=$ 0.2 in an arbitrary direction misaligned with the orbital angular momentum. We will refer to this case as $S_{0.20}^{0.99}$, using a notation similar to that introduced earlier. The simulation has $M \omega_{\text {orb }}=0.0148$ at $t=t_{\text {relax }}$, and then proceeds through 23 orbits, merger, and ringdown. This simulation took approximately 26 days on 48 cores for the highest resolution using the same optimized version of SpEC as the $S_{0.994}^{++}$case described in Sec. III B.

We found that for $S_{0.20}^{0.99}$ we needed to remove the effect of unresolved initial transients and increase the spacing in AMR truncation error tolerance to obtain acceptable convergence results. To do this we followed the same procedure as for $S_{0.994}^{++}$, described in Sec. III B. Figure 7 shows good convergence of the gravitational-wave phase difference when using this procedure.

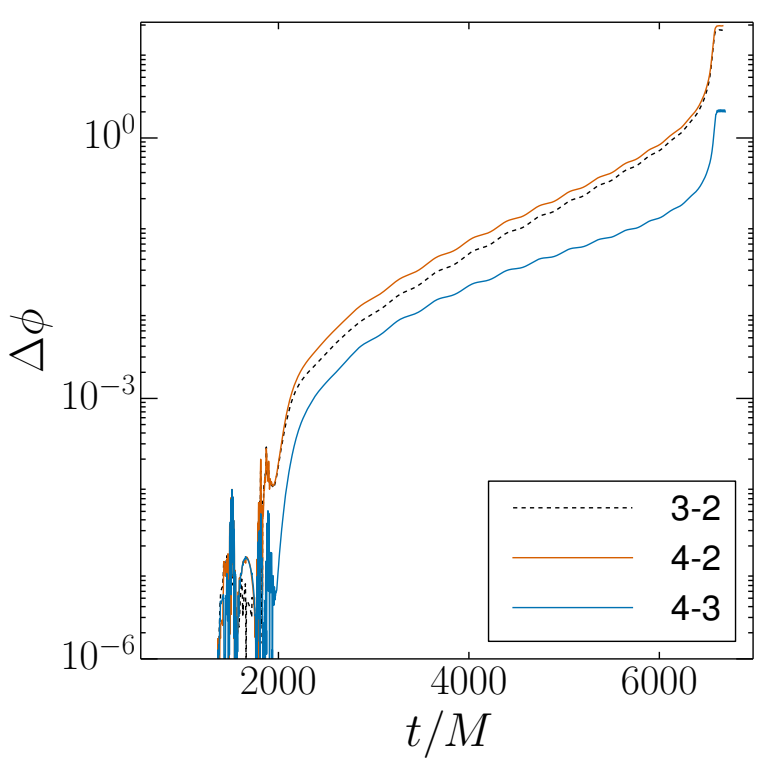

FIG. 7. Convergence test for $S_{0.20}^{0.99}$. Labels are the same as for Figure 4. For $N \neq 4$, the simulations were started at $t_{\text {branch }}=1362 M$, using the $N=4$ solution as initial data.

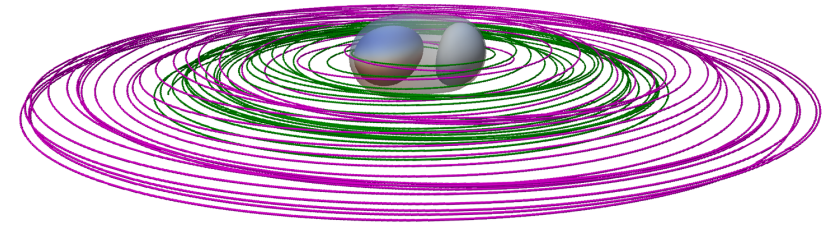

FIG. 8. Coordinate trajectories (green and purple lines) of the black holes and coordinate shapes of the individual and common apparent horizons (surfaces) at the moment of merger, for $S_{0.20}^{0.99}$. The horizons are colored according to their vorticity [79].

Figure 8 shows the trajectories of the centers of the apparent horizons for this simulation, as well as the individual apparent horizons and the common apparent horizon at the moment when the common horizon first appears. Trajectories and horizon shapes are shown in the asymptotically inertial coordinate system used in the simulation. Because the spin of the smaller hole $\vec{\chi}_{B}$ is not aligned with the orbital angular momentum, the system precesses, so the trajectories do not lie in a plane.

Figure 9 shows the precession of the spin and orbital frequency vectors in $S_{0.20}^{0.99}$. The spin $\vec{\chi}_{A}$ and orbital frequency $\vec{\omega}_{\text {orb }}$ initially point along the $z$-axis. Because the misaligned spin $\vec{\chi}_{B}$ is on the smaller black hole and is much smaller in magnitude than $\vec{\chi}_{A}$, it has a minimal effect on the orbital dynamics, so $\vec{\chi}_{A}$ and $\vec{\omega}_{\text {orb }}$ remain near the $z$-axis throughout the simulation. Therefore, we consider the precession to be mild. As angular momentum is carried away by gravitational radiation, the opening angles of the precession cones change. The an- 


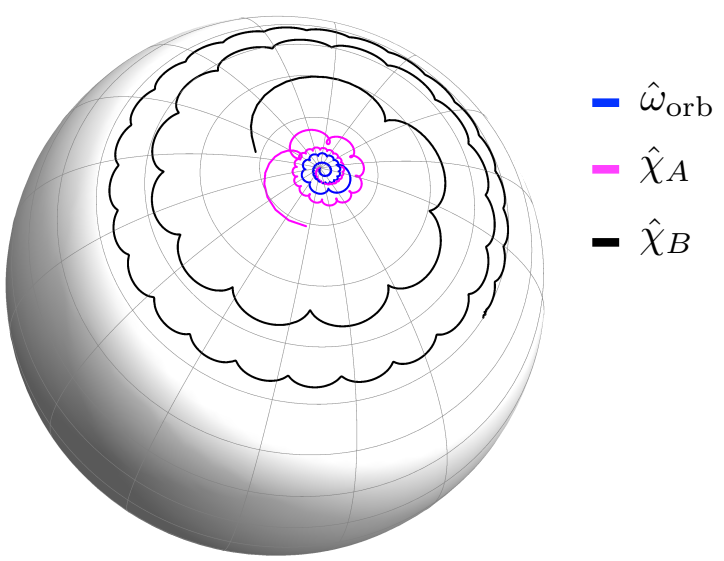

FIG. 9. Precession of the spins and orbital frequency for the highest-resolution simulation $N=4$ of $S_{0.20}^{0.99}$. The unit vector spins, $\hat{\chi}_{A}$ and $\hat{\chi}_{B}$, and orbital frequency $\hat{\omega}_{\text {orb }}$ trace the precession on the unit sphere. The precession curves for $N=$ 2 and $N=3$ converge to the $N=4$ curves shown here, and the curves for $N=3$ and $N=4$ are nearly indistinguishable.

gles of $\vec{\omega}_{\text {orb }}$ and $\vec{\chi}_{A}$ with respect to the $z$-axis increase from $0^{\circ}$ at $t=0$ to $6^{\circ}$ and $12^{\circ}$, respectively, at the time of merger. In contrast, the angle of $\vec{\chi}_{B}$ with respect to the $z$-axis decreases from $45^{\circ}$ to $12^{\circ}$. The spins $\vec{\chi}_{A}$ and $\vec{\chi}_{B}$ complete 2.1 and 2.5 precession cycles, respectively, and $\vec{\omega}_{\text {orb }}$ completes 2.4 precession cycles.

The spin of the remnant black hole is $\chi_{f}=0.89692(5)$, and the radiated energy fraction is $E_{\mathrm{rad}}=7.8560(8) \%$. The formulas from Healy et al. (2014) [25] predict $\chi_{f}=0.89686$ and $E_{\text {rad }}=7.8365 \%$. Even though these predictions lie outside the numerical uncertainty of the measured values, the agreement is quite good. ${ }^{3}$

\section{RESULTS}

\section{A. Spin evolution during inspiral}

During the inspiral, the tidal field of each black hole affects its companion, and this interaction slowly changes the black-hole masses and spins as a function of time. For aligned spins, Alvi [78] has derived perturbative expressions for the time rate of change of the mass and spin of a black hole in a binary. Chatziioannou, Poisson,

3 To evaluate the quantities $S_{\|}$and $\Delta_{\|}$in Ref. [25], we used the $z$-component of $\vec{S}$ and $\vec{\Delta}$ at $t_{\text {relax }}$, which should be strictly valid only for non-precessing binaries. Also, the formula for $\chi_{f}$ in Ref. [25] requires evaluating certain quantities at the innermost stable circular orbit (ISCO) of a Kerr black hole with a spin of $\chi_{f}$, so that $\chi_{f}$ is not given in closed form; for simplicity we evaluate the ISCO quantities using the measured $\chi_{f}$ from the simulation.

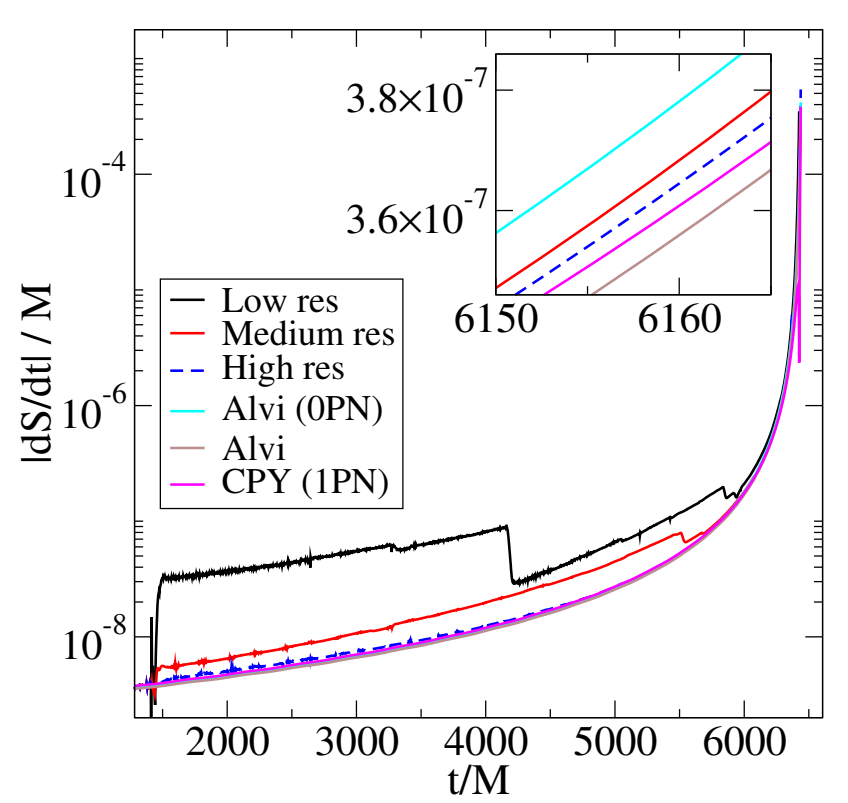

FIG. 10. Magnitude of $d S / d t$ of one of the black holes from $S_{0.994}^{++}$. Shown are three numerical resolutions, Alvi's expression as written (Eq. (11) of [78]), Alvi's expression truncated to leading order, and the CPY expression[80, 81] to $1 \mathrm{PN}$ order. The inset zooms closer to the high-resolution numerical curve.

and Yunes (hereafter CPY) [80], have recently extended these expressions to higher order in perturbation theory. Although CPY's expressions in Ref. [80] are computed to $1.5 \mathrm{PN}$ beyond leading order (i.e. terms in $d S / d t$ proportional to $v^{15}$ and terms in $d M / d t$ proportional to $v^{18}$, where $v^{2}=M / r$ is the $\mathrm{PN}$ expansion parameter), their 1.5PN terms are incorrect and will be corrected soon [81]; so here we will truncate CPY's expressions to 1PN order.

In our simulations we track the apparent horizons as a function of time, and at frequent time intervals we measure both the surface area and the spin of the horizons. The spin computation is carried out using the approximate Killing vector formalism of Cook, Whiting, and Owen [71, 72]. The mass of the black hole is then computed using Christodoulou's formula. We compare our numerical results to the analytic results of Alvi and CPY.

To compare a black-hole mass or spin from a numerical simulation to that of a perturbative expression, the two quantities must be compared at the same event along the black hole trajectory. Although waveform quantities at future null infinity computed by numerical simulations are routinely compared with waveforms computed by PN expansions, it is not straightforward to compare nearzone quantities like black-hole masses and spins because of gauge ambiguities. Here we make two comparisons. The first compares quantities at the same numerical and perturbative $t$ coordinate. The second assumes that the orbital angular velocity $\omega_{\text {orb }}=d \phi / d t$ of the black hole in the numerical simulation can be equated with that of the perturbative expression. Note that in both the nu- 


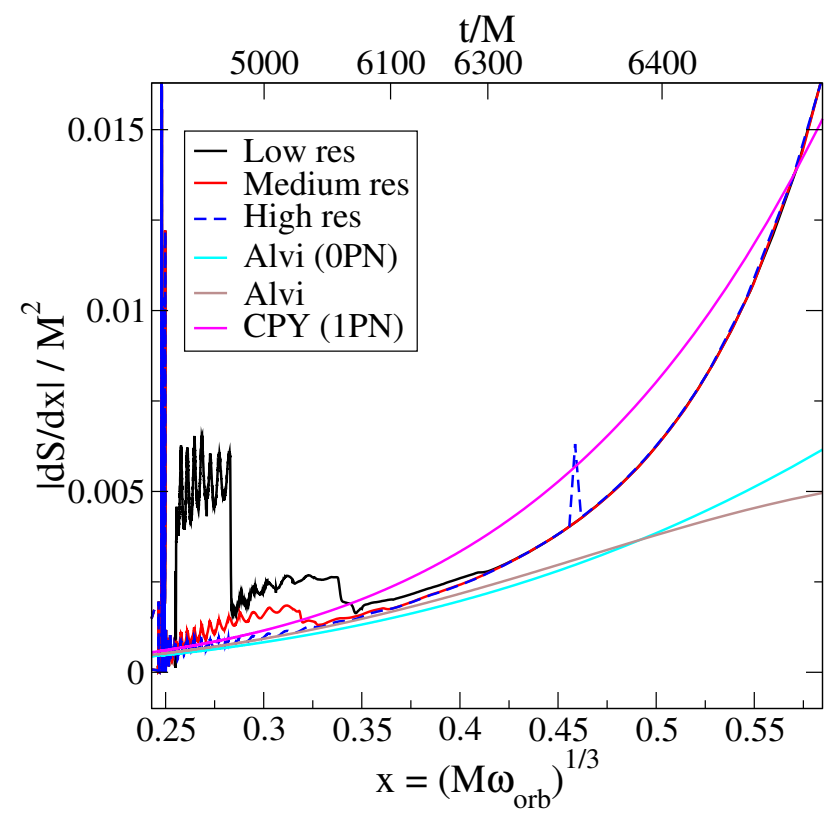

FIG. 11. Magnitude of $d S / d x$, where $x \equiv\left(M \omega_{\text {orb }}\right)^{1 / 3}$, of one of the black holes from $S_{0.994}^{++}$. The top horizontal axis shows $t / M$ of the highest-resolution numerical simulation, for comparison with values of $x$.

merical and perturbative cases, the $t$ coordinate becomes the Minkowski $t$ at infinity, and the $\phi$ coordinate is periodic. Because of the approximate helical Killing vector $d / d t+\omega_{\text {orb }} d / d \phi, \omega_{\text {orb }}$ is approximately an angular velocity at infinity. Therefore, one might hope that equating the perturbative and numerical $\omega_{\text {orb }}$ yields better agreement than, e.g., equating the radial coordinate $r$ of the simulation with that of perturbation theory.

Figure 10 compares the magnitude of $d S / d t$ of one of the black holes for $S_{0.994}^{++}$with the expressions of both CPY and Alvi. We include numerical results for three resolutions in Fig. 10 because the magnitude of $d S / d t$ is extremely small and difficult to resolve. Indeed, the lowest resolution fails to resolve $d S / d t$ until around $t=6000 M$, when $d S / d t$ grows to about $10^{-7} M$, and the medium resolution fails to resolve $d S / d t$ only slightly earlier. Note that Alvi's expression includes some 1.5PN terms, but ignores $1 \mathrm{PN}$ effects such as magnetic-type tidal perturbations and the difference between the global PN time coordinate and the local time coordinate of a frame moving along with one of the black holes. Therefore, we plot both Alvi's expression in its entirety, and Alvi's expression truncated to lowest $(0 \mathrm{PN})$ order. The CPY expression includes $0 \mathrm{PN}$ and $1 \mathrm{PN}$ terms. The CPY and Alvi expressions agree to $0 \mathrm{PN}$ order.

Figure 10 shows overall excellent agreement between the PN and numerical simulation results. All the perturbative curves agree within our our numerical error up to $t \sim 6100 M$, but for $t>6150 M$ none of the perturbative approximations agree with the numerical result within numerical error. This disagreement at late

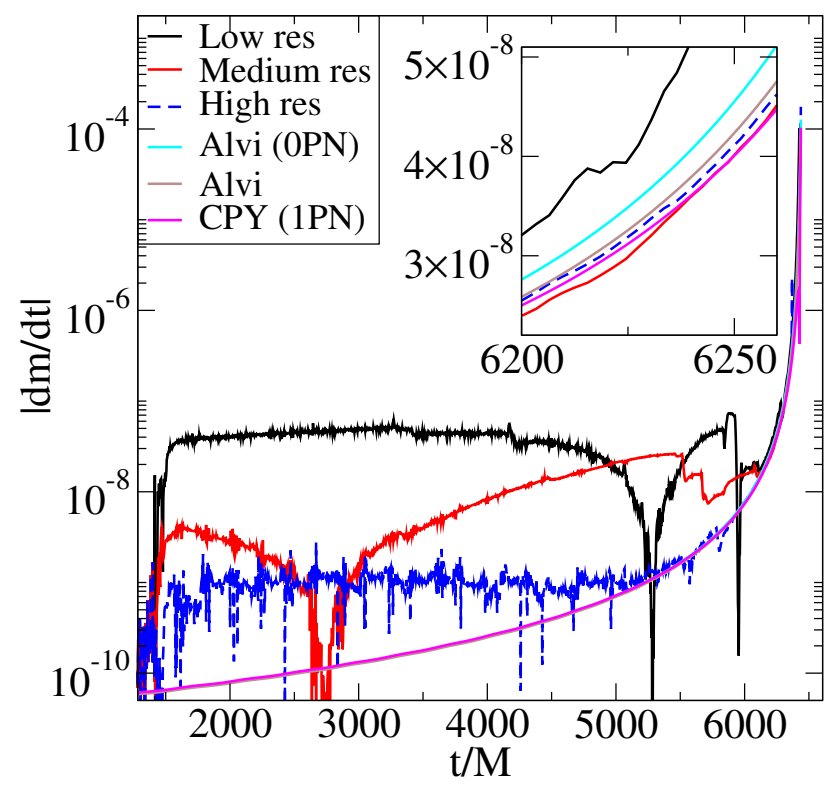

FIG. 12. Magnitude of $d m / d t$ of one of the black holes from $S_{0.994}^{++}$. Shown are three numerical resolutions, Alvi's expression as written (Eq. (11) of [78]), Alvi's expression truncated to leading order, and the CPY expression[80, 81] truncated to $1 \mathrm{PN}$ order. The inset zooms closer to the high-resolution numerical curve.

times is not surprising since all the perturbative expressions should lose accuracy shortly before merger. We can eliminate the time coordinate, a possible source of gauge dependence, by instead plotting $d S / d x$ versus $x$, where $x \equiv\left(M \omega_{\text {orb }}\right)^{1 / 3}$. This is shown in Fig. 11. To obtain $d S / d x$ from $d S / d t$ and to obtain $x$ from $t$, it is necessary to have some function $x(t)$. For the numerical curves, this function is obtained from the numerical time coordinate and the numerical orbital frequency. For the perturbative curves, this function is the PN expression for $x(t)$ derived from Eq. (4.14) of Ref. [82]. Thus, all the numerical curves in Figs. 10 and 11 are independent of any perturbative assumptions, and all the perturbative curves in Figs. 10 and 11 are independent of the numerical data, except that the perturbative and numerical $t$ coordinates are both represented by the same horizontal axis of Fig. 10, and the perturbative and numerical $\omega_{\text {orb }}$ are both represented by the same horizontal axis of Fig. 11.

In Fig. 11, the perturbative and numerical expressions agree early in the inspiral, but not at late times; this is expected because perturbative expressions become inaccurate for large $x$. Alvi's full expression appears to agree with the numerical simulations slightly better than the others for small $x$, but that expression diverges from the numerical result at larger $x$ earlier than the others. Note that Fig. 11 emphasizes late times because the frequency increases very rapidly with time.

Figures 12 and 13 are similar to Figs. 10 and 11 except that they show the change in Christodoulou mass instead of the change in dimensionful spin. As was the case for 


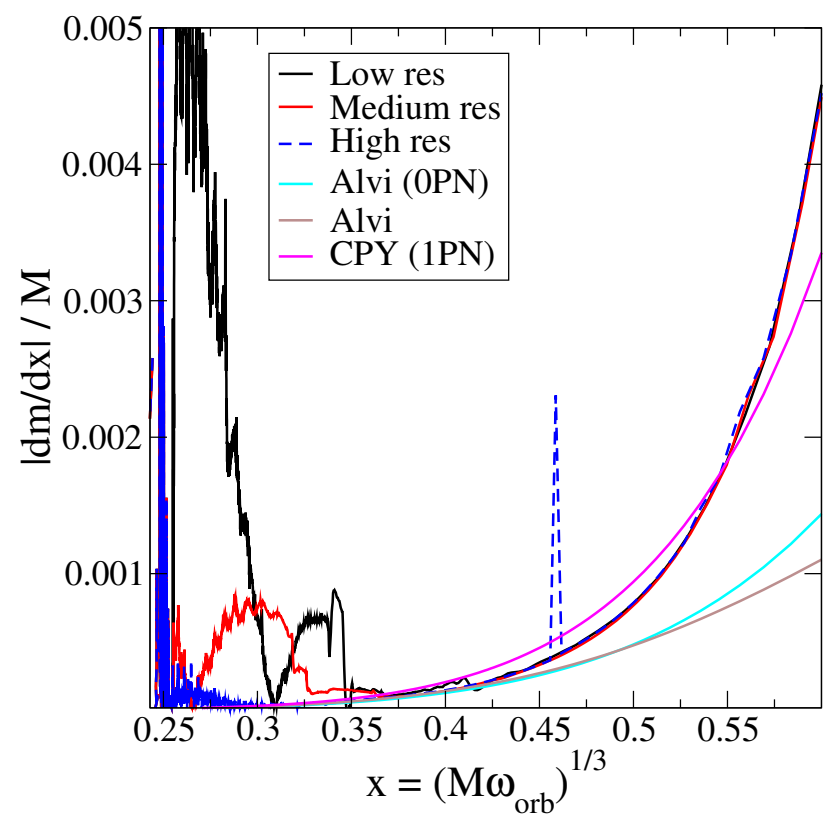

FIG. 13. Magnitude of $d m / d x$, where $x \equiv\left(M \omega_{\text {orb }}\right)^{1 / 3}$, of one of the black holes from $S_{0.994}^{++}$.

the spin comparisons, both the Alvi and CPY formulas agree well with each other and with the numerical result early in the inspiral, but do not agree at late times. Note that since the derivative of the mass is smaller (by a factor of $v^{3}$ in $\mathrm{PN}$ ) than the derivative of the spin, $d m / d t$ is more difficult to resolve numerically than $d S / d t$, as seen by the larger numerical errors in Figs. 12 and 13 compared with the numerical errors in Figs. 10 and 11.

\section{B. Orbital hangup}

During a BBH inspiral, the orbital frequency $\omega_{\text {orb }}$ secularly evolves along with the black-hole masses and spins. For equal-mass binaries with equal spins aligned (or antialigned) with the orbital angular momentum, the number of orbits until merger increases as a function of $S \cdot L$. Damour [83] observed this effect, today commonly called "orbital hangup", in an effective-one-body model of the holes' motion; the effect is a consequence of postNewtonian spin-orbit coupling [84]. Campanelli, Lousto, and Zlochower [85] first demonstrated orbital hangup in numerical simulations of merging BBHs.

Instead of examining the number of orbits from the trajectories, we infer the number of orbits from the dominant $\ell=m=2$ mode of the emitted gravitational waves ${ }^{4}$. We do this because it is easier to define a gauge-invariant

\footnotetext{
${ }^{4}$ Specifically, we extrapolate the gravitational waves measured on a series of concentric shells to $r \rightarrow \infty$, as discussed in detail in Sec. IV C.
}

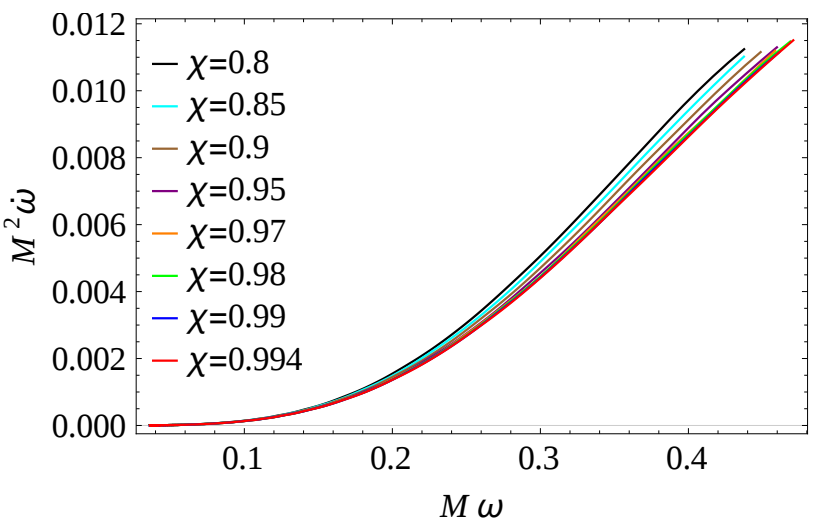

FIG. 14. The evolution of the derivative of the gravitationalwave frequency $\dot{\omega}_{22}=d \omega_{22} / d t$, for simulations $S_{0.99}^{++}$and $S_{0.994}^{++}$and (for comparison) simulations $S_{0.8}^{++}$[41], $S_{0.85}^{++}$[41], $S_{0.9}^{++}[41], S_{0.95}^{++}[41], S_{0.97}^{++}[37]$, and $S_{0.98}^{++}[24]$.

time of merger from the waveforms than from the trajectories; specifically, we define the time of merger as the time when the waveform amplitude is at a maximum.

Let $h_{22}(t)$ be the ${ }_{-2} Y_{22}$ spin-weighted spherical harmonic mode of the gravitational wave strain $h(t)$, and let $\omega_{22}$ be the frequency of $h_{22}(t)$. Figure 14 shows the time evolution of $d \omega_{22} / d t$ for simulations $S_{0.99}^{++}$and $S_{0.994}^{++}$. For comparison, we also show results for other simulations with equal masses and equal spins aligned with the orbital angular momentum [24, 37, 41]. Note that $d \omega_{22} / d t$ is positive and steadily increasing: the frequency does not slow down or momentarily remain constant, as a literal interpretation of the term "orbital hangup" might suggest.

Figure 15 shows the gravitational-wave cycles accumulated between an initial gravitational-wave frequency of $M \omega_{22}=0.036$ (i.e., an initial orbital frequency of $M \omega_{\text {orb }}=0.018$ ) and merger (when the amplitude of $h_{22}$ peaks). Simulations $S_{0.99}^{++}$and $S_{0.994}^{++}$reveal that the orbital hangup depends approximately linearly on the initial spin $\chi$, even at spins that are nearly extremal; however, most of our simulations only agree with the linear fit to $\mathcal{O}(0.1 \%)$, which is often larger than our estimated numerical uncertainties. This linearity implies that even near extremality, the orbital hangup effect is dominated by spin-orbit coupling; resolving nonlinear features in Fig. 15 would require more simulations with higher accuracy.

\section{Comparison with analytic approximants}

We compare the gravitational waveforms from our simulations to several analytic waveform approximants. The numerical waveforms were computed by performing Regge-Wheeler-Zerilli extraction [86, 87] at a sequence of radii between $100 M$ and $465 M$, and then extrapolating to $\mathscr{I}^{+}$using the open-source GWFrames 

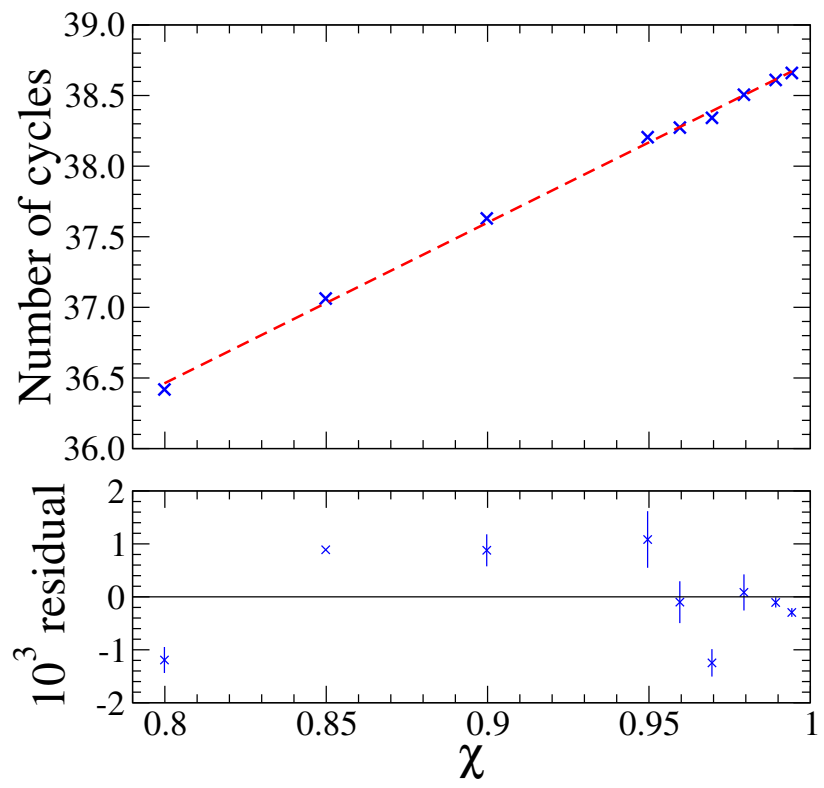

FIG. 15. The number of gravitational-wave cycles as a function of the initial spin $\chi$, measured after the initial relaxation, for simulations $S_{0.99}^{++}$and $S_{0.994}^{++}$and (for comparison) simulations $S_{0.8}^{++}$[41], $S_{0.85}^{++}$[41], $S_{0.9}^{++}$[41], $S_{0.95}^{++}$[41], $S_{0.96}^{++}$[50], $S_{0.97}^{++}$[37], and $S_{0.98}^{++}$[24]. Upper panel: The number of gravitational-wave cycles of $h_{22}$ accumulated between a gravitational-wave frequency $M \omega_{22}=0.036$ and merger (i.e., the time when the amplitude of $h_{22}$ peaks). The dashed line is a linear fit to the data. Lower panel: Fractional difference ("residual") between our results and the linear fit, with uncertainties for simulations except $S_{0.85}^{++}$(which we ran at only one resolution) estimated as differences between medium and high numerical resolutions.

software package [88-90]. The TaylorT1, TaylorT4, and TaylorT5 approximants were constructed using the PostNewtonian module in GWFrames. ${ }^{5}$ The EOB approximants were constructed using SEOBNRv2 [49] from the LIGO Algorithm Library, with the function SimIMRSpinAlignedEOBWaveform modified to return $h_{22}(t)$. Physical parameters for the approximants were taken from the highest resolution from each simulation at the relaxation time. Because SEOBNRv2 is strictly valid only for non-precessing systems, and therefore accepts only scalar values of the spins as input, it is not obvious what to input for the case of $S_{0.20}^{0.99}$. We pass the $z$-component of the spins into SimIMRSpinAlignedEOBWaveform. If instead we pass the

\footnotetext{
5 To our knowledge, the PostNewtonian module includes all terms currently found in the literature. Non-spin terms are given up to 4.0 PN order for the binding energy [13, 91]; $3.5 \mathrm{PN}$ with incomplete 4.0 PN information for the flux [13]; and 3.5 PN for the waveform modes [92-94]. The spin-orbit terms are given to 4.0 $\mathrm{PN}$ in the binding energy [95]; $3.5 \mathrm{PN}$ with incomplete 4.0 $\mathrm{PN}$ terms in flux [96]; and 2.0 PN in the waveform modes [89]. Terms quadratic in spin are given to $2.0 \mathrm{PN}$ order in the binding energy and flux [97, 98], and waveform modes [89, 97, 99].
}

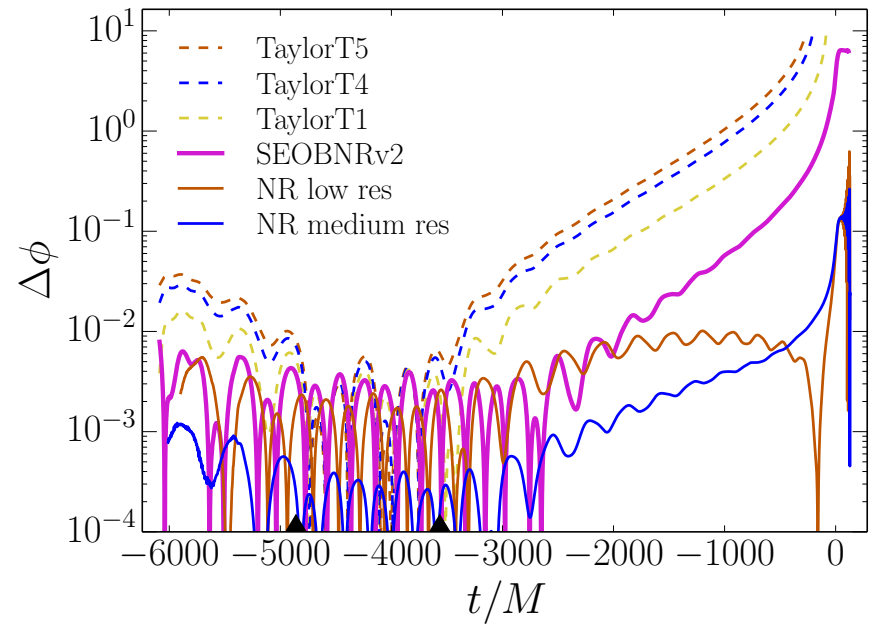

FIG. 16. Phase differences $\Delta \phi$ of $h_{22}$ as a function of retarded time before merger for $S_{0.99}^{++}$. Shown are differences between the highest numerical resolution and several analytic approximants. Differences between the highest numerical resolution and other numerical resolutions are shown for comparison. The waveforms are aligned in the time interval delimited by the black triangles.

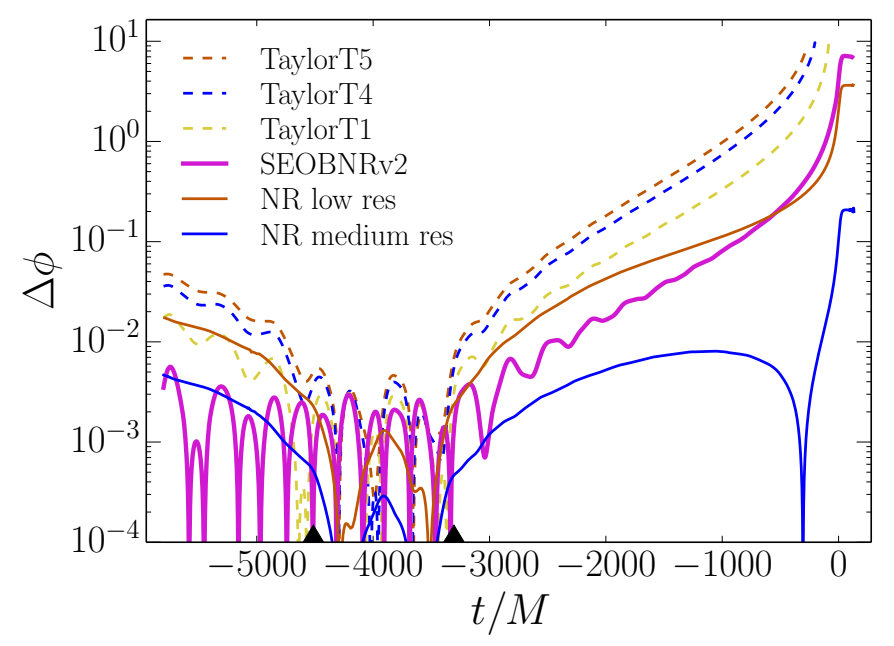

FIG. 17. Phase differences $\Delta \phi$ of $h_{22}$ between numerical and approximant data for $S_{0.994}^{++}$. Labels are the same as for Fig. 16.

spin magnitudes, we see larger disagreements between the EOB and numerical waveforms for $S_{0.20}^{0.99}$, likely due to a change in the strength of spin-orbit coupling. We will see below that non-precessing EOB agrees remarkably well with $S_{0.20}^{0.99}$ despite the mild precession of this simulation.

In Figs. 16, 17, and 18, we show for $S_{0.99}^{++}, S_{0.994}^{++}$, and $S_{0.20}^{0.99}$ (respectively) the phase difference $\Delta \phi$ of $h_{22}$ between the highest numerical resolution and the PN and EOB approximants. We also include $\Delta \phi$ between the highest numerical resolution and other numerical resolutions for comparison. To compute $\Delta \phi$, we first align each waveform with the highest resolution numericalrelativity (NR) waveform using the procedure prescribed 


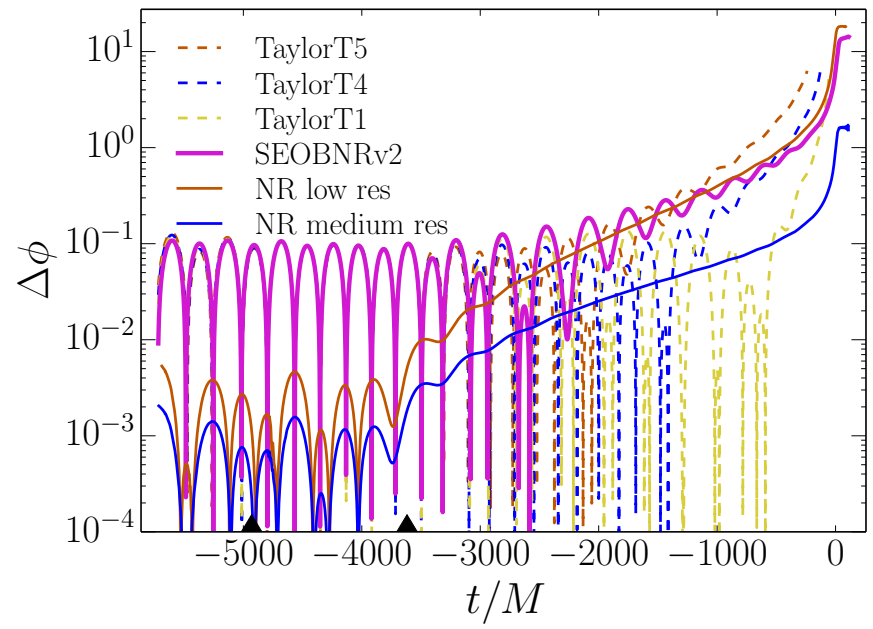

FIG. 18. Phase differences $\Delta \phi$ of $h_{22}$ between numerical and approximant data for $S_{0.20}^{0.99}$. Labels are the same as for Fig. 16 . Note that the Taylor models include precession but SEOBNRv2 does not. However, the precession of $S_{0.20}^{0.99}$ is mild so the numerical waveform still agrees reasonably well with SEOBNRv2.

in Ref. [100]: we find the time offset $\delta t$ and phase offset $\delta \phi$ that minimize $\Phi(\delta t, \delta \phi)$, a measure of the phase difference in $h_{22}$, given by

$$
\Phi(\delta t, \delta \phi) \equiv \int_{t_{1}}^{t_{2}}\left[\phi_{a}(t)-\phi_{b}(t+\delta t)+\delta \phi\right]^{2} d t,
$$

where $\delta \phi$ can be computed analytically from $\delta t$

$$
\delta \phi(\delta t)=\frac{1}{t_{2}-t_{1}} \int_{t_{1}}^{t_{2}}\left[\phi_{a}(t)-\phi_{b}(t+\delta t)\right] d t .
$$

The alignment interval $t \in\left[t_{1}, t_{2}\right]$ is the same for all comparisons with a particular simulation. The lower bound $t_{1}$ is chosen such that the junk radiation has left the computational domain for all numerical resolutions, specifically $t_{1}=\max \left[t_{0}+3\left(t_{\text {relax }}-t_{0}\right)\right]$, where $t_{0}$ is the time at the beginning of the waveform. The upper bound $t_{2}$ is chosen such that the gravitational-wave frequency changes by at least $10 \%$ during the interval $\left[t_{1}, t_{2}\right]$, as suggested in Ref. [101].

We have also computed $\Delta \phi$ with a few other alignment methods, including the three-dimensional minimization of complex $h_{22}$ differences in Ajith et al. 2008 (Eq. 4.9 in Ref. [102]) and the four-dimensional minimization over time and frame-rotation degrees of freedom in Boyle 2013 (Eq. 22 in Ref. [88]). We have found that our results are qualitatively independent of alignment method.

The TaylorT family of PN approximants shows the largest discrepancy with our highest numerical resolution. Phase errors between PN and NR waveforms grow to several radians before the merger in every case. The smallest phase errors outside the alignment interval occur for $S_{0.20}^{0.99}$, which is likely a consequence of the smaller black hole having a moderate spin. We find the best agreement with TaylorT1, in contrast to PN comparisons for other nearly extremal systems [37], which found the best agreement with TaylorT4 for spins aligned with the orbital angular momentum; note that the PN waveforms considered in Ref. [37] include fewer higher-order PN terms than we do here. This is further evidence that agreement with a particular PN approximant in the TaylorT family depends sensitively on the PN order. Agreement with a particular PN approximant also depends on the parameters of the simulation (e.g., Ref. [37]).

The EOB approximant performs significantly better than the PN approximants for $S_{0.99}^{++}$and $S_{0.994}^{++}$, which is impressive considering that the parameters of these waveforms are outside the range in which SEOBNRv2 was calibrated to NR. Only about 5 radians of phase error is accumulated in $S_{0.99}^{++}$and $S_{0.994}^{++}{ }^{6}$ Phase error increases to a little over 10 radians in $S_{0.20}^{0.99}$, but this case is precessing, and SEOBNRv2 is only valid for non-precessing systems. However, the precession is mild (cf. Figs. 8 and 9), which could account for the relatively good agreement.

The analytic approximants show much larger $\Delta \phi$ at early times for $S_{0.20}^{0.99}$ (see Fig. 18) than for $S_{0.99}^{++}$and $S_{0.994}^{++}$. We conjecture that this is due to the relatively large eccentricity of $S_{0.20}^{0.99}$ (see Table I), whereas the PN and EOB models used here are non-eccentric. Note that we use precessing PN models for comparing to $S_{0.20}^{0.99}$.

The phase errors between numerical waveforms computed at different resolutions are convergent. Because of the rapid convergence, the difference between the two highest numerical resolutions represents the numerical error in the second highest resolution; to determine the numerical error of the highest resolution waveform, we would need to perform a simulation at an even higher resolution. As a conservative estimate of the numerical error of the highest resolution waveform, we use the difference between the two highest-resolution waveforms as an upper bound. The upper bound of the numerical phase error of the highest resolution simulation, computed in this way, is thus about 0.2 radians for $S_{0.99}^{++}$and $S_{0.994}^{++}$and about 1 radian for $S_{0.20}^{0.99}$.

In Figs. 16, 17, and 18, the larger numerical phase errors in the lower resolutions of $S_{0.994}^{++}$and $S_{0.20}^{0.99}$ are expected, because these simulations use a larger spacing in AMR truncation error tolerance as described in Sec. III B. The larger spacing increases relative phase errors between successive numerical resolutions. Nevertheless, our comparisons show that numerical errors are much smaller than the errors in the PN and EOB waveforms for systems with nearly extremal black holes, indicating that these numerical waveforms will be useful for calibrating and extending the regime of validity for approximate waveforms.

\footnotetext{
${ }^{6}$ Note that SEOBNRv2 was calibrated by minimizing unfaithfulness rather than phase error; it is possible to have relatively large phase errors even when the unfaithfulness is small [49].
} 


\section{CONCLUSION}

We have presented improved methods for simulating the binary evolution of nearly extremal black holes, i.e., black holes with spins above the Bowen-York limit of $\chi=0.93$. These techniques enable robust simulations in the portion of $\mathrm{BBH}$ parameter space where the black holes have very large spins. Because nearly extremal black holes might exist in astrophysical binaries, these simulations will be important for helping to maximize what we can learn from gravitational-wave experiments.

We have applied our new methods to carry out the first unequal-mass, mildly-precessing $\mathrm{BBH}$ simulation containing a nearly extremal black hole, and to extend aligned-spin BBH simulations to spin magnitudes that begin to approach the Novikov-Thorne limit of $\chi=0.998$. From these new simulations, we have learned that perturbative predictions for tidal heating and tidal torquing agree well with the numerics at low frequency, even for nearly extremal spins. However, we find that our numerical errors are still large enough that we cannot reliably distinguish between $0 \mathrm{PN}$ and $1 \mathrm{PN}$ predictions. Doing so would require further investigation with more accurate simulations.

While many physical quantities depend on $\chi$ in an extremely nonlinear fashion, we find that the number of orbits starting from a chosen orbital frequency (i.e., the orbital hangup) scales approximately linearly with $\chi$. Finally, after demonstrating numerical convergence, we have found that our numerical waveforms agree with SEOBNRv2 much better than with TaylorT PN approximants, even though the parameters for these simulations are outside the range in which SEOBNRv2 was calibrated. However, even the SEOBNRv2 waveforms disagree with our numerical waveforms by more than our numerical truncation error. This indicates that these simulations are sufficiently accurate to validate and further improve analytical waveform approximants for future gravitationalwave observations. How significant these improvements will be for Advanced LIGO is the subject of future work.

\section{ACKNOWLEDGMENTS}

We are grateful to Eric Poisson, Nicolas Yunes, and Katerina Chatziioannou for detailed discussions about perturbative expressions for tidal torquing and about the problems inherent in comparing numerical and postNewtonian expressions for near-field quantities. We thank Alessandra Buonanno and Sebastiano Bernuzzi for helpful discussions. Simulations used in this work were computed with SpEC [47]. This work was supported in part by the Sherman Fairchild Foundation; NSF grants PHY-1440083 and AST-1333520 at Caltech, NSF grants PHY-1306125 and AST-1333129 at Cornell, and NSF grant PHY-1307489 at California State University Fullerton; a 2013-2014 California State University Fullerton Junior Faculty Research Grant. Computations were performed on the Zwicky cluster at Caltech, which is supported by the Sherman Fairchild Foundation and by NSF award PHY-0960291; on the NSF XSEDE network under grant TG-PHY990007N; on the Orca cluster supported by NSF award NSF-1429873 and by California State University Fullerton; and on the GPC supercomputer at the SciNet HPC Consortium [103]. SciNet is funded by: the Canada Foundation for Innovation under the auspices of Compute Canada; the Government of Ontario; Ontario Research Fund-Research Excellence; and the University of Toronto.
[1] G. M. Harry (LIGO Scientific Collaboration), Class. Quant. Grav. 27, 084006 (2010).

[2] The Virgo Collaboration, "Advanced Virgo Baseline Design," (2009), [VIR-0027A-09].

[3] The Virgo Collaboration, "Advanced Virgo Technical Design Report," (2012), [VIR-0128A-12].

[4] K. Somiya and the KAGRA Collaboration, Class. Quantum Grav. 29, 124007 (2012).

[5] L. Gou, J. E. McClintock, M. J. Reid, J. A. Orosz, J. F. Steiner, R. Narayan, J. Xiang, R. A. Remillard, K. A. Arnaud, and S. W. Davis, Astrophys. J. 742, 85 (2011), arXiv:1106.3690 [astro-ph.HE].

[6] A. Fabian, D. Wilkins, J. Miller, R. Reis, C. Reynolds, et al., MNRAS 424, 217 (2012), arXiv:1204.5854 [astroph.HE].

[7] L. Gou, J. E. McClintock, R. A. Remillard, J. F. Steiner, M. J. Reid, et al., Astrophys.J. 790, 29 (2014).

[8] J. E. McClintock, R. Shafee, R. Narayan, R. A. Remillard, S. W. Davis, and L.-X. Li, Astrophys. J. 652, 518 (2006).

[9] J. Miller, C. Reynolds, A. Fabian, G. Miniutti, and
L. Gallo, Astrophys.J. 697, 900 (2009), arXiv:0902.2840 [astro-ph.HE].

[10] D. Walton, E. Nardini, A. Fabian, L. Gallo, and R. Reis, MNRAS 428, 2901 (2013), arXiv:1210.4593 [astro-ph.HE].

[11] J. E. McClintock, R. Narayan, and J. F. Steiner, Space Sci.Rev. 183, 295 (2014), arXiv:1303.1583 [astroph.HE].

[12] C. S. Reynolds, (2013), arXiv:1302.3260 [astro-ph.HE].

[13] L. Blanchet, Living Rev.Rel. 9, 4 (2006).

[14] F. Pretorius, Phys. Rev. Lett. 95, 121101 (2005), arXiv:gr-qc/0507014 [gr-qc].

[15] M. Campanelli, C. Lousto, P. Marronetti, and Y. Zlochower, Phys. Rev. Lett. 96, 111101 (2006), arXiv:grqc/0511048 [gr-qc].

[16] J. G. Baker, J. Centrella, D.-I. Choi, M. Koppitz, and J. van Meter, Phys. Rev. Lett. 96, 111102 (2006), arXiv:gr-qc/0511103 [gr-qc].

[17] J. Centrella, J. G. Baker, B. J. Kelly, and J. R. van Meter, Rev. Mod. Phys. 82, 3069 (2010).

[18] H. P. Pfeiffer, Class. Quant. Grav. 29, 124004 (2012), 
arXiv:1203.5166 [gr-qc].

[19] M. Hannam, (2013), arXiv:1312.3641 [gr-qc].

[20] A. Le Tiec, Int. J. Mod. Phys. D 23, 1430022 (2014), arXiv:1408.5505 [gr-qc].

[21] P. Ajith, M. Boyle, D. A. Brown, B. Brugmann, L. T. Buchman, et al., Class. Quantum Grav. 29, 124001 (2012).

[22] I. Hinder et al. (The NRAR Collaboration), Classical and Quantum Gravity 31, 025012 (2014), arXiv:1307.5307 [gr-qc].

[23] L. Pekowsky, R. O'Shaughnessy, J. Healy, and D. Shoemaker, Phys. Rev. D 88, 024040 (2013), arXiv:1304.3176 [gr-qc].

[24] A. H. Mroue, M. A. Scheel, B. Szilagyi, H. P. Pfeiffer, M. Boyle, D. A. Hemberger, L. E. Kidder, G. Lovelace, S. Ossokine, N. W. Taylor, A. Zenginoglu, L. T. Buchman, T. Chu, E. Foley, M. Giesler, R. Owen, and S. A. Teukolsky, Phys. Rev. Lett. 111, 241104 (2013), arXiv:1304.6077 [gr-qc].

[25] J. Healy, C. O. Lousto, and Y. Zlochower, Phys. Rev. D 89, 104052 (2014), arXiv:1406.7295 [gr-qc].

[26] J. Clark, L. Cadonati, J. Healy, I. S. Heng, J. Logue, et al., (2014), arXiv:1406.5426 [gr-qc].

[27] J. M. Bowen, Gen. Relativ. Gravit. 11, 227 (1979).

[28] J. M. Bowen and J. W. York, Jr., Phys. Rev. D 21, 2047 (1980).

[29] S. Brandt and B. Brügmann, Phys. Rev. Lett. 78, 3606 (1997).

[30] J. W. York, Jr., in Essays in General Relativity, edited by F. J. Tipler (Academic, New York, 1980) pp. 39-58.

[31] A. Garat and R. H. Price, Phys. Rev. D 61, 124011 (2000).

[32] J. A. Valiente Kroon, Phys. Rev. Lett. 92, 041101 (2004).

[33] G. B. Cook and J. W. York, Jr., Phys. Rev. D 41, 1077 (1990).

[34] S. Dain, C. O. Lousto, and R. Takahashi, Phys. Rev. D 65, 104038 (2002).

[35] M. Hannam, S. Husa, and N. O. Murchadha, Phys. Rev. D 80, 124007 (2009).

[36] G. Lovelace, M. A. Scheel, and B. Szilágyi, Phys. Rev. D 83, 024010 (2011), arXiv:1010.2777 [gr-qc].

[37] G. Lovelace, M. Boyle, M. A. Scheel, and B. Szilágyi, Class. Quant. Grav. 29, 045003 (2012), arXiv:arXiv:1110.2229 [gr-qc].

[38] G. Lovelace, M. D. Duez, F. Foucart, L. E. Kidder, H. P. Pfeiffer, M. A. Scheel, and B. Szilágyi, Class. Quantum Grav. 30, 135004 (2013), arXiv:1302.6297 [gr-qc].

[39] I. D. Novikov and K. S. Thorne, "Black holes," (Gordon and Breach, New York, 1973) p. 343.

[40] K. S. Thorne, Astrophys. J. 191, 507 (1974).

[41] D. A. Hemberger, G. Lovelace, T. J. Loredo, L. E. Kidder, M. A. Scheel, B. Szilágyi, N. W. Taylor, and S. A. Teukolsky, Phys. Rev. D 88, 064014 (2013), arXiv:1305.5991 [gr-qc].

[42] L. Rezzolla et al., Astrophys. J. 679, 1422 (2008), arXiv:0708.3999 [gr-qc].

[43] M. Hannam, S. Husa, F. Ohme, D. Müller, and B. Brügmann, Phys. Rev. D 82, 124008 (2010), arXiv:1007.4789.

[44] P. Marronetti, W. Tichy, B. Brügmann, J. González, and U. Sperhake, Phys. Rev. D 77, 064010 (2008).

[45] S. Dain, C. O. Lousto, and Y. Zlochower, Phys. Rev. D 78, 024039 (2008), arXiv:0803.0351v2 [gr-qc].
[46] I. Ruchlin, J. Healy, C. O. Lousto, and Y. Zlochower, (2014), arXiv:1410.8607 [gr-qc].

[47] http://www.black-holes.org/SpEC.html.

[48] G. Lovelace, R. Owen, H. P. Pfeiffer, and T. Chu, Phys. Rev. D 78, 084017 (2008).

[49] A. Taracchini, A. Buonanno, Y. Pan, T. Hinderer, M. Boyle, D. A. Hemberger, L. E. Kidder, G. Lovelace, A. H. Mroue, H. P. Pfeiffer, M. A. Scheel, B. Szilagyi, and A. Zenginoglu, Phys.Rev. D89, 061502 (2014), arXiv:1311.2544 [gr-qc].

[50] G. Lovelace, M. A. Scheel, R. Owen, M. Giesler, R. Katebi, B. Szilágyi, T. Chu, N. Demos, D. A. Hemberger, L. E. Kidder, H. P. Pfeiffer, and N. Afshari, (2014), submitted to Class. Quantum Grav., arXiv:1411.7297 [gr-qc].

[51] H. P. Pfeiffer, L. E. Kidder, M. A. Scheel, and S. A. Teukolsky, Comput. Phys. Commun. 152, 253 (2003).

[52] M. Caudill, G. B. Cook, J. D. Grigsby, and H. P. Pfeiffer, Phys. Rev. D 74, 064011 (2006), gr-qc/0605053.

[53] J. W. York, Phys. Rev. Lett. 82, 1350 (1999).

[54] H. P. Pfeiffer, D. A. Brown, L. E. Kidder, L. Lindblom, G. Lovelace, and M. A. Scheel, Class. Quantum Grav. 24, S59 (2007), gr-qc/0702106.

[55] A. Buonanno, L. E. Kidder, A. H. Mroué, H. P. Pfeiffer, and A. Taracchini, Phys. Rev. D 83, 104034 (2011), arXiv:1012.1549 [gr-qc].

[56] A. H. Mroué and H. P. Pfeiffer, (2012), arXiv:1210.2958 [gr-qc].

[57] H. Friedrich, Commun. Math. Phys. 100, 525 (1985).

[58] D. Garfinkle, Phys. Rev. D 65, 044029 (2002).

[59] F. Pretorius, Class. Quantum Grav. 22, 425 (2005).

[60] L. Lindblom, M. A. Scheel, L. E. Kidder, R. Owen, and O. Rinne, Class. Quantum Grav. 23, S447 (2006).

[61] L. Lindblom and B. Szilágyi, Phys. Rev. D 80, 084019 (2009), arXiv:0904.4873.

[62] M. W. Choptuik and F. Pretorius, Phys. Rev. Lett. 104, 111101 (2010), arXiv:0908.1780 [gr-qc].

[63] B. Szilágyi, L. Lindblom, and M. A. Scheel, Phys. Rev. D 80, 124010 (2009), arXiv:0909.3557 [gr-qc].

[64] B. Szilágyi, Int.J.Mod.Phys. D23, 1430014 (2014), arXiv:1405.3693 [gr-qc].

[65] M. A. Scheel, M. Boyle, T. Chu, L. E. Kidder, K. D. Matthews and H. P. Pfeiffer, Phys. Rev. D 79, 024003 (2009), arXiv:gr-qc/0810.1767.

[66] D. A. Hemberger, M. A. Scheel, L. E. Kidder, B. Szilágyi, G. Lovelace, N. W. Taylor, and S. A. Teukolsky, Class. Quantum Grav. 30, 115001 (2013), arXiv:1211.6079 [gr-qc].

[67] S. Ossokine, L. E. Kidder, and H. P. Pfeiffer, arXiv:1304.3067 (2013), arXiv:1304.3067 [gr-qc].

[68] O. Rinne, Class. Quantum Grav. 23, 6275 (2006).

[69] O. Rinne, L. Lindblom, and M. A. Scheel, Class. Quantum Grav. 24, 4053 (2007).

[70] C. Gundlach, Phys. Rev. D 57, 863 (1998).

[71] G. B. Cook and B. F. Whiting, Phys. Rev. D 76, 041501(R) (2007).

[72] R. Owen, Topics in Numerical Relativity: The periodic standing-wave approximation, the stability of constraints in free evolution, and the spin of dynamical black holes, Ph.D. thesis, California Institute of Technology (2007).

[73] M. A. Scheel, H. P. Pfeiffer, L. Lindblom, L. E. Kidder, O. Rinne, and S. A. Teukolsky, Phys. Rev. D 74, 104006 (2006). 
[74] M. Boyle, D. A. Brown, L. E. Kidder, A. H. Mroué, H. P. Pfeiffer, M. A. Scheel, G. B. Cook, and S. A. Teukolsky, Phys. Rev. D 76, 124038 (2007), arXiv:0710.0158 [grqc].

[75] L. T. Buchman, H. P. Pfeiffer, M. A. Scheel, and B. Szilágyi, Phys. Rev. D 86, 084033 (2012), arXiv:1206.3015 [gr-qc].

[76] T. Chu, H. P. Pfeiffer, and M. A. Scheel, Phys. Rev. D 80, 124051 (2009), arXiv:0909.1313 [gr-qc].

[77] http://www.black-holes.org/waveforms.

[78] K. Alvi, Phys. Rev. D 64, 104020 (2001).

[79] R. Owen, J. Brink, Y. Chen, J. D. Kaplan, G. Lovelace, K. D. Matthews, D. A. Nichols, M. A. Scheel, F. Zhang, A. Zimmerman, and K. S. Thorne, Phys. Rev. Lett. 106, 151101 (2011).

[80] K. Chatziioannou, E. Poisson, and N. Yunes, Phys. Rev. D 87, 044022 (2013), arXiv:1211.1686 [gr-qc].

[81] K. Chatziioannou, E. Poisson, and N. Yunes, In preparation.

[82] L. E. Kidder, Phys. Rev. D 52, 821 (1995).

[83] T. Damour, Phys. Rev. D 64, 124013 (2001), arXiv:grqc/0103018 [gr-qc].

[84] L. E. Kidder, Phys. Rev. D52, 821 (1995), arXiv:grqc/9506022.

[85] M. Campanelli, C. O. Lousto, and Y. Zlochower, Phys. Rev. D 74, 041501(R) (2006), gr-qc/0604012.

[86] O. Sarbach and M. Tiglio, Phys. Rev. D 64, 084016 (2001).

[87] O. Rinne, L. T. Buchman, M. A. Scheel, and H. P. Pfeiffer, Class. Quantum Grav. 26, 075009 (2009).

[88] M. Boyle, Phys. Rev. D 87, 104006 (2013).

[89] M. Boyle, L. E. Kidder, S. Ossokine, and H. P. Pfeiffer, (2014), arXiv:1409.4431, arXiv:1409.4431.

[90] S. Ossokine, M. Boyle, L. E. Kidder, A. H. Mroué, H. P. Pfeiffer, M. A. Scheel, and B. Szilágyi, (2014), in preparation.

[91] D. Bini and T. Damour, "Analytical determination of the two-body gravitational interaction potential at the 4th post-Newtonian approximation," (2013), arXiv:1305.4884 [gr-qc].

[92] L. Blanchet, G. Faye, B. R. Iyer, and S. Sinha, Class. Quant. Grav. 25, 165003 (2008), arXiv:0802.1249 [grqc].

[93] G. Faye, S. Marsat, L. Blanchet, and B. R. Iyer, Class. Quant. Grav. 29, 175004 (2012).

[94] G. Faye, L. Blanchet, and B. R. Iyer, "Non-linear multipole interactions and gravitational-wave octupole modes for inspiralling compact binaries to third-and-ahalf post-Newtonian order," (2014), arXiv:1409.3546 [gr-qc].

[95] A. Bohé, S. Marsat, G. Faye, and L. Blanchet, Class. Quant. Grav. 30, 075017 (2013).

[96] S. Marsat, L. Blanchet, A. Bohé, and G. Faye, "Gravitational waves from spinning compact object binaries: New post-Newtonian results," (2013), arXiv:1312.5375 [gr-qc].

[97] C. M. Will and A. G. Wiseman, Phys. Rev. D 54, 4813 (1996).

[98] K. Arun, A. Buonanno, G. Faye, and E. Ochsner, Phys. Rev. D 79, 104023 (2009), arXiv:0810.5336 [gr-qc].

[99] A. Buonanno, G. Faye, and T. Hinderer, Phys. Rev. D 87, 044009 (2013).

[100] M. Boyle, A. Buonanno, L. E. Kidder, A. H. Mroué, Y. Pan, et al., Phys. Rev. D 78, 104020 (2008), arXiv:0804.4184 [gr-qc].

[101] I. MacDonald, S. Nissanke, and H. P. Pfeiffer, Class. Quantum Grav. 28, 134002 (2011), arXiv:1102.5128 [grqc].

[102] P. Ajith, S. Babak, Y. Chen, M. Hewitson, B. Krishnan, et al., Phys. Rev. D 77, 104017 (2008), arXiv:0710.2335 [gr-qc].

[103] C. Loken, D. Gruner, L. Groer, R. Peltier, N. Bunn, M. Craig, T. Henriques, J. Dempsey, C.-H. Yu, J. Chen, L. J. Dursi, J. Chong, S. Northrup, J. Pinto, N. Knecht, and R. V. Zon, J. Phys.: Conf. Ser. 256, 012026 (2010). 